\title{
Four-Week Repeated Intravenous Dose Toxicity and Toxicokinetic Study of TS-DP2, a Novel Human Granulocyte Colony Stimulating Factor in Rats
}

\author{
JooBuom Lee ${ }^{1,4}$, Kyungsun Lee², Keunbum Choe ${ }^{1,2}$, Hyunseob Jung ${ }^{2}$, Hyunseok Cho², Kiseok Choi' ${ }^{2}$, Taegon Kim², \\ Seojin Kim ${ }^{2}$, Hyeong-Seok Lee ${ }^{3}$, Mi-Jin $\mathrm{Cha}^{3}$, Si-Whan Song ${ }^{3}$, Chul Kyu Lee ${ }^{3 \dagger}$ and Gie-Taek Chun ${ }^{4}$ \\ ${ }^{1}$ Bio Business Department, TS Corporation, Korea \\ ${ }^{2} R \& D$ Center, TS Corporation, Korea \\ ${ }^{3}$ Nonclinical Research Institute, Chemon Inc., Korea \\ ${ }^{4}$ Department of Molecular Bioscience, College of Bio Medical Science, Kangwon National University, Korea
}

(Received June 4, 2015; Revised August 22, 2015; Accepted October 12, 2015)

\begin{abstract}
TS-DP2 is a recombinant human granulocyte colony stimulating factor (rhG-CSF) manufactured by TS Corporation. We conducted a four-week study of TS-DP2 (test article) in repeated intravenous doses in male and female Sprague-Dawley (SD) rats. Lenograstim was used as a reference article and was administered intravenously at a dose of $1000 \mu \mathrm{g} / \mathrm{kg} /$ day. Rats received TS-DP2 intravenously at doses of 250, 500, and $1000 \mu \mathrm{g} / \mathrm{kg} /$ day once daily for 4 weeks, and evaluated following a 2-week recovery period. Edema in the hind limbs and loss of mean body weight and body weight gain were observed in both the highest dose group of TS-DP2 and the lenograstim group in male rats. Fibro-osseous lesions were observed in the lenograstim group in both sexes, and at all groups of TS-DP2 in males, and at doses of TS-DP2 $500 \mu \mathrm{g} / \mathrm{kg} /$ day and higher in females. The lesion was considered a toxicological change. Therefore, bone is the primary toxicological target of TS-DP2. The lowest observed adverse effect level (LOAEL) in males was $250 \mu \mathrm{g} / \mathrm{kg} /$ day, and no observed adverse effect level (NOAEL) in females was $250 \mu \mathrm{g} / \mathrm{kg} / \mathrm{day}$ in this study. In the toxicokinetic study, the serum concentrations of G-CSF were maintained until $8 \mathrm{hr}$ after administration. The systemic exposures $\left(\mathrm{AUC}_{0-24 \mathrm{~h}}\right.$ and $\left.\mathrm{C}_{0}\right)$ were not markedly different between male and female rats, between the administration periods, or between TS-DP2 and lenograstim. In conclusion, TS-DP2 shows toxicological similarity to lenograstim over 4-weeks of repeated doses in rats.
\end{abstract}

Key words: Neutropenia, rhG-CSF, TS-DP2, Lenograstim, Toxicity, Toxicokinetics, Rats

\section{INTRODUCTION}

The two hematopoietic growth factors used clinically are recombinant human (rh) granulocyte colony-stimulating factor (G-CSF, filgrastim [Neupogen]) and granulocytemacrophage colony-stimulating factor (GM-CSF, sargramostim [Leukine]). These factors differ significantly in their role in hematopoiesis and the regulation of mature effector cell function. G-CSF regulates neutrophil production and and release of neutrophils from the marrow in response to infection. It can also regulate release of neutrophils from the

Correspondence to: Chul Kyu Lee, Nonclinical Research Institute, Chemon Inc., 15F, Gyeonggi Bio Center, 147, Gwanggyo-ro, Yeongtong-gu, Suwon-si, Gyeonggi-do 443-766, Korea

E-mail: cklee@chemon.co.kr

This is an Open-Access article distributed under the terms of the Creative Commons Attribution Non-Commercial License (http:// creativecommons.org/licenses/by-nc/3.0) which permits unrestricted non-commercial use, distribution, and reproduction in any medium, provided the original work is properly cited. marrow in response to infection. Conversely, GM-CSF mediates its effects on neutrophils via phagocytic accessory cells and synergy with G-CSF, but it does not appear to have a role in basal hematopoiesis (1).

Endogenous G-CSF levels rise during neutropenia or infection, and neutrophils are able to regulate G-CSF levels via a feedback mechanism (2). G-CSF induces activation and priming of neutrophils in several in vitro assays via functional receptors, and increases neutrophil migration to tissues without increased adhesion to endothelial surfaces (3). G-CSF is also an antiapoptotic factor that prolongs neutrophil survival. There are currently two "unmodified" forms of rhG-CSF worldwide in clinical use: filgrastim, which is expressed in Escherichia coli, and lenograstim, which is expressed in Chinese hamster ovary (CHO) cells and is only available outside of the United States. Following subcutaneous administration, rhG-CSF has a terminal serum half-life of approximately $2 \sim 5 \mathrm{hr}$, and confers a doserelated increase in circulating neutrophils. RhG-CSF causes increased neutrophil production, release from the marrow, 
and increased survival $(4,5)$. Neutrophils produced in response to rhG-CSF therapy function normally or "supernormally" in in-vitro function assays (6). Toxicities of rhGCSF include bone pain and rare cases of Sweet's syndrome (neutrophilic dermatosis). Several cases of steroid-responsive interstitial pulmonary infiltrates have been reported in rhG-CSF-treated patients with hematologic malignancies (7). Pegylated filgrastim (pegfilgrastim, Neulasta ${ }^{\circledR}$ ) is formulated to have a prolonged serum half-life (particularly in neutropenic patients) and appears to be cleared by neutrophils and their marrow precursors (8). Pegfilgrastim does not appear to be associated with any additional toxicities or an increase in the incidence or severity of bone pain.

TS-DP2, manufactured by TS Corporation, is a novel biosimilar candidate of lenograstim. Therefore, it is necessary to prove toxicological similarity with a contemporary G-CSF for the investigational new drug (IND) application to the Korea FDA. In this study, we used lenograstim (Neutrogin injection, JW Pharmaceutical) as a reference article for comparison of TS-DP2 toxicity and toxicokinetic properties.

\section{MATERIALS AND METHODS}

Materials. TS-DP2 $(250 \mu \mathrm{g} / \mathrm{mL} ; 50 \mathrm{~mL} / \mathrm{vial}), \mathrm{TS}-\mathrm{DP} 2$ dilution buffer, lenograstim $(250 \mu \mathrm{g} /$ vial, Lot. No. 13008, white freeze-dried powder or chunks in a colorless vial), and the water for injection (WFI) for dilution of lenograstim were supplied from TS Co.

Animal care and maintenance. Specific Pathogen Free (SPF) male and female SD rats were purchased from Koatech Co. Ltd. (Pyeongtaek-si, Republic of Korea) and allowed a week of quarantine and acclimatization. Environmental conditions in the barrier animal facility area were maintained as following: $23 \pm 3^{\circ} \mathrm{C}, 55 \pm 15 \%$ relative humidity, 10 20 times/h ventilation frequency, 150 300 Lux of luminous intensity, and 12-hr light/12-hr dark cycle. Animals were offered the irradiation-sterilized pellet food (Teklad rodent diet 2918C, Harlan Co. Ltd., USA). Underground water disinfected by ultraviolet sterilizer and ultrafiltration was given via water bottle, ad libitum. This study was approved by the Institutional Animal Care and Use Committee (IACUC) in Chemon Inc. (Serial No. 14-R106 for the general toxicity study and No. 14-R271 for toxicokinetic study).

Preparation of TS-DP2. The high dose tested utilized the original provided solution without dilution. The original solution was diluted with a provided dilution buffer in a serial manner for the middle and low doses. The formulation was prepared by adding $1 \mathrm{~mL}$ WFI into the original vial to create a final concentration of $250 \mu \mathrm{g} / \mathrm{mL}$. After the dose formulation, it was filtered using a $0.2 \mu \mathrm{m}$ DVDF syringe filter. On the first day of dosing, $\mathrm{pH}$ values of each solution were measured using a $\mathrm{pH}$ meter (Orion 3 Star, Thermo, USA) were $6.50,6.54$, and 6.56 for the low, middle, and high doses, respectively. The formulations were prepared daily for immediate use.

Dosing and grouping. The animal was placed in restraints and the appropriate solution administered via intravenous infusion into the lateral tail veins using a disposable syringe $(3.0 \mathrm{~mL}, 26 \mathrm{G})$ at a rate of approximately $4 \mathrm{~mL} / \mathrm{min}$. The first day of dosing was designated as Study Day 1. Study animals were divided into the groups listed in the table below (Table 1).

Body weight. Animals were observed three times daily for clinical signs. Body weight of each rat was recorded weekly, starting on Study Day 1 to the end of the study (necropsy). The body weight at the conclusion of the study was measured after fasting overnight.

Food and water consumption. Food and water consumption were measured weekly and calculated to the mean daily consumption per animal. Ophthalmological examination was performed at the last week of observation in 5 animals from each sex in the main study group and recovery group $(n=20)$.

Urinalysis. In the last week of observation, 5 animals from each sex of the main study group and recovery group

Table 1. Group assignment

\begin{tabular}{cccccccc}
\hline \hline Group & $\begin{array}{c}\text { Total number of } \\
\text { Animal/Sex }\end{array}$ & $\begin{array}{c}\text { Dose } \\
(\mu \mathrm{g} / \mathrm{kg} / \text { day })\end{array}$ & $\begin{array}{c}\text { Day 29 } \\
\text { Main Sacrifice }\end{array}$ & $\begin{array}{c}\text { Day 43 } \\
\text { Recovery Sacrifice }\end{array}$ & Toxicokinetics & $\begin{array}{c}\text { Dose volume } \\
(\mathrm{mL} / \mathrm{kg})\end{array}$ & $\begin{array}{c}\text { Formulation } \\
\text { Conc. }(\mu \mathrm{g} / \mathrm{mL})\end{array}$ \\
\hline $\mathrm{G} 1$ & $18 \mathrm{M} / 18 \mathrm{~F}$ & 0 & $10 \mathrm{M} / 10 \mathrm{~F}$ & $5 \mathrm{M} / 5 \mathrm{~F}$ & $3 \mathrm{M} / 3 \mathrm{~F}$ & 4 & 0 \\
$\mathrm{G} 2$ & $21 \mathrm{M} / 21 \mathrm{~F}$ & 1000 & $10 \mathrm{M} / 10 \mathrm{~F}$ & $5 \mathrm{M} / 5 \mathrm{~F}$ & $6 \mathrm{M} / 6 \mathrm{~F}$ & 4 & 250 \\
$\mathrm{G} 3$ & $16 \mathrm{M} / 16 \mathrm{~F}$ & 250 & $10 \mathrm{M} / 10 \mathrm{~F}$ & 0 & $6 \mathrm{M} / 6 \mathrm{~F}$ & 4 & 62.5 \\
$\mathrm{G} 4$ & $16 \mathrm{M} / 16 \mathrm{~F}$ & 500 & $10 \mathrm{M} / 10 \mathrm{~F}$ & 0 & $6 \mathrm{M} / 6 \mathrm{~F}$ & 4 & 125 \\
$\mathrm{G} 5$ & $21 \mathrm{M} / 21 \mathrm{~F}$ & 1000 & $10 \mathrm{M} / 10 \mathrm{~F}$ & $5 \mathrm{M} / 5 \mathrm{~F}$ & $6 \mathrm{M} / 6 \mathrm{~F}$ & 4 & 250 \\
\hline
\end{tabular}

$\mathrm{M}=$ Male, $\mathrm{F}=$ Female.

G1: Negative control group (WFI, JW Pharmaceutical), G2: Reference control group (Lenograstim), G3-G5: Test article treatment group (TSDP2). 
were housed separately in a metabolic cage for urine collection. The urinalysis (general urine examination and urine sediment test) was performed using $1 \mathrm{~mL}$ freshly collected urine sample, and the total volume of urine collected for $24 \mathrm{hr}$ was measured. Urine was analyzed with an automatic analyzer (Clinitek Advantus, Siemens, USA) and a test strip (Multistix 10SG, SIEMENS, USA). Urinalysis parameters were glucose, $\mathrm{pH}$, bilirubin, protein, ketone body, urobilinogen, occult blood, nitrite, clarity, specific gravity, and urine color. Urine sediments (white blood cell, epithelial cells, red blood cell, cast) were examined by microscope after S-M (Sternheimer-Malbin) dying.

Hematological test. Blood samples were collected from the posterior vena cava of all animals scheduled for necropsy under deep isoflurane (Ifran liquid, Hana Pharm. Co., Ltd.) anesthesia. Approximately $1 \mathrm{~mL}$ blood taken during the necropsy was placed into a CBC bottle (Vacutainer $3 \mathrm{~mL}, \mathrm{BD}, \mathrm{USA})$ that contained anticoagulant EDTA-2K. Hematological parameters were determined using a Coulter counter (Advia 2120, Siemens, USA), and included the following: red blood cell (RBC), red cell distribution width (RDW), lymphocyte (LYM), hemoglobin concentration (HGB), hemoglobin distribution width (HDW), monocyte (MONO), hematocrit (HCT), platelet count (PLT), eosinophil (EOS), mean corpuscular volume (MCV), mean platelet volume (MPV), basophil (BASO), mean corpuscular hemoglobin (MCH), white blood cell (WBC), large unstained cell (LUC), mean corpuscular hemoglobin concentration (MCHC), neutrophil (NEU), and reticulocytes (RET). Blood coagulation times (activated partial thromboplastin time (APTT) and prothrombin time (PT)) were measured by the nephelometric analysis method with a coagulation time analyzer (ACL 100, Instrumentation Laboratory, USA) and reported in seconds.

Serum biochemistry. A portion of the collected blood was placed into a 5-mL vacutainer tube (Insepack, Sekisui, Japan) that contained a clot activator. The blood was coagulated at room temperature for 15 20 min, and then centrifuged (3000 RPM, 1902 RCF, Combi-514R, Hanil, Korea) for $10 \mathrm{~min}$. Serum biochemical parameters including aspartate aminotransferase (AST), total cholesterol (TCHO), creatinine (CRE), alanine aminotransferase (ALT), triglyceride (TG), inorganic phosphorus (IP), alkaline phosphatase (ALP), total protein (TP), calcium ion $\left(\mathrm{Ca}^{2+}\right)$, creatine phosphokinase (CPK), albumin (ALB), potassium ion $\left(\mathrm{K}^{+}\right)$, total bilirubin (TBIL), albumin/globulin ratio (A/G), sodium ion $\left(\mathrm{Na}^{+}\right)$, glucose (GLU), blood urea nitrogen (BUN), and chloride ion $\left(\mathrm{Cl}^{-}\right)$were measured by using AU680 serum biochemistry analyzer (Beckman Coulter, USA).

Organ weights. Absolute weight and relative organ-tobody weight ratios were determined after sacrifice at 4 weeks. Tissues included brain, spleen, epididymis, pituitary gland, adrenal gland, prostate gland, lung, kidney, ovary, heart, liver, uterus, thymus, and testis.

Gross findings and histopathology. At the scheduled termination, the organs and tissues in the cranial, thoracic, and abdominal cavities of euthanized rats underwent gross examination for visible defects. Each organ was excised and fixed in $10 \%$ neutral buffered formalin, except in Davidson's fixative for the eye and in Bouin's fixative for testis and epididymis. Fixed organs were embedded in paraffin, and microsections taken at $4 \sim 5 \mu \mathrm{m}$ thickness. Hematoxylin $\&$ eosin-stained slides were prepared, and the specimens were microscopically examined with an optical microscope. Gradation of lesions was scaled from 1 to 5 according on Pristima ${ }^{\circledR}$ program. In general, a minimal lesion was +1 and massive lesion was +5 . Two between changes in direct proportion to the evenly divided into five portions, were carried out in a semi-quantitative.

Toxicokinetics (TK) analysis. On Day 1 and 28, blood samples of approximately $0.5 \mathrm{~mL}$ were collected from the jugular vein from 6 animals from each sex in the TS-DP2 group and 3 animals from each sex from the lenograstim group. The blood samples were stored at room temperature in a dark place for approximately $30 \mathrm{~min}$, and then centrifuged at $3000 \mathrm{~g}\left(4^{\circ} \mathrm{C}\right)$ for approximately $20 \mathrm{~min}$. The resulting serum samples were transferred to two clean labeled tubes and stored in a deep freezer (below $-70^{\circ} \mathrm{C}$ ) until analysis. Sample tubes were labeled with study no., period, group, animal ID, and blood collection time.

Blood collection times for TK study were pre-dose, $0.5 \mathrm{hr}$, and $24 \mathrm{hr}$ after administration for G1 (negative control) and pre-dose, $5 \mathrm{~min}, 0.5 \mathrm{hr}, 1 \mathrm{hr}, 2 \mathrm{hr}, 4 \mathrm{hr}, 8 \mathrm{hr}$, and $24 \mathrm{hr}$ after administration for G2-G5 (lenograstim and TS-DP2 groups), respectively. Quantification of G-CSF in rat serum was performed by the following validated method: 'Analytical method validation of G-CSF in rat serum using ELISA (Chemon Study No.: 13-RV-557)'. The analytical batch consisted of a blank, calibration samples (8 concentrations), quality control (QC) samples (low, medium, and high levels), and study samples. Calibration samples should have relative error (RE) within $\pm 20 \%$ LLOQ (lower limit of quantification) and ULOQ (upper limit of quantification) within $\pm 25 \%$, and at least $75 \%$ of calibration samples must fulfill this criterion. QC samples should have RE within $\pm 20 \%$ at each level, and at least $67 \%$ of QC samples must fulfill this criterion. Sample re-analysis was performed if an analytical batch failed or obtained concentration is above the ULOQ. Incurred sample reanalysis (ISR) samples were randomly selected, and comprised $10 \%$ of the entire study samples at around $\mathrm{C}_{\max }$ and in the elimination phase. The variability within $\pm 20 \%$, and at least $67 \%$ of ISR samples must fulfill this criterion. ISR was satisfied with acceptance 
Table 2. Clinical signs in Sprague-Dawley rats intravenously treated with TS-DP2 for 4 weeks

\begin{tabular}{|c|c|c|c|c|c|c|c|c|c|c|c|c|c|}
\hline \multirow{2}{*}{ Sex } & \multirow{2}{*}{ Treatment } & \multirow{2}{*}{$\begin{array}{c}\text { Dosage } \\
(\mu \mathrm{g} / \mathrm{kg} / \text { day })\end{array}$} & \multirow{2}{*}{ Signs } & \multicolumn{10}{|c|}{ Days } \\
\hline & & & & $1 \sim 20$ & 21 & 22 & 23 & 24 & 25 & 26 & 27 & 28 & 29 \\
\hline \multirow{9}{*}{ Male } & PBS & Control & Normal & $15 / 15$ & $15 / 15$ & $15 / 15$ & $15 / 15$ & $15 / 15$ & $15 / 15$ & $15 / 15$ & $15 / 15$ & $15 / 15$ & $15 / 15$ \\
\hline & Lenograstim & 1000 & Normal & $15 / 15$ & $15 / 15$ & $13 / 15$ & $11 / 15$ & $11 / 15$ & $10 / 15$ & $10 / 15$ & $9 / 15$ & $8 / 15$ & $8 / 15$ \\
\hline & & & Edema & $15 / 15$ & $15 / 15$ & $2 / 15$ & $4 / 15$ & $4 / 15$ & $5 / 15$ & $5 / 15$ & $6 / 15$ & $7 / 15$ & $7 / 15$ \\
\hline & TS-D & 250 & Normal & $10 / 10$ & $10 / 10$ & $10 / 10$ & $10 / 10$ & $10 / 10$ & $10 / 10$ & $10 / 10$ & $10 / 10$ & $10 / 10$ & $10 / 10$ \\
\hline & & 500 & Normal & $10 / 10$ & $10 / 10$ & $10 / 10$ & $10 / 10$ & $10 / 10$ & $10 / 10$ & $10 / 10$ & $10 / 10$ & $10 / 10$ & $10 / 10$ \\
\hline & & 1000 & Normal & $14 / 15$ & $13 / 15$ & $13 / 15$ & $11 / 15$ & $11 / 15$ & $11 / 15$ & $11 / 15$ & $9 / 15$ & $9 / 15$ & $9 / 15$ \\
\hline & & & Edema & $1 / 15$ & $2 / 15$ & $2 / 15$ & $4 / 15$ & $4 / 15$ & $4 / 15$ & $4 / 15$ & $6 / 15$ & $6 / 15$ & $6 / 15$ \\
\hline & & & Abnormal gait & $0 / 15$ & $0 / 15$ & $0 / 15$ & $0 / 15$ & $0 / 15$ & $0 / 15$ & $0 / 15$ & $0 / 15$ & $0 / 15$ & $0 / 15$ \\
\hline & & & Ataxia & $1 / 15$ & $1 / 15$ & $1 / 15$ & $1 / 15$ & $1 / 15$ & $1 / 15$ & $1 / 15$ & $1 / 15$ & $1 / 15$ & $1 / 15$ \\
\hline \multirow{8}{*}{ Female } & PBS & Control & Normal & $15 / 15$ & $15 / 15$ & $15 / 15$ & $15 / 15$ & $15 / 15$ & $15 / 15$ & $15 / 15$ & $15 / 15$ & $15 / 15$ & $15 / 15$ \\
\hline & Lenograstim & 1000 & Normal & $15 / 15$ & $15 / 15$ & $14 / 15$ & $14 / 15$ & $14 / 15$ & $14 / 15$ & $14 / 15$ & $14 / 15$ & $13 / 15$ & $14 / 15$ \\
\hline & & & Edema & $15 / 15$ & $15 / 15$ & $1 / 15$ & $1 / 15$ & $1 / 15$ & $1 / 15$ & $1 / 15$ & $1 / 15$ & $2 / 15$ & $1 / 15$ \\
\hline & TS-DP2 & 250 & Normal & $10 / 10$ & $10 / 10$ & $10 / 10$ & $10 / 10$ & $10 / 10$ & $10 / 10$ & $10 / 10$ & $10 / 10$ & $10 / 10$ & $10 / 10$ \\
\hline & & 500 & Normal & $10 / 10$ & $9 / 10$ & $9 / 10$ & $9 / 10$ & $9 / 10$ & $9 / 10$ & $9 / 10$ & $9 / 10$ & $9 / 10$ & $9 / 10$ \\
\hline & & & Edema & $10 / 10$ & $1 / 10$ & $1 / 10$ & $1 / 10$ & $1 / 10$ & $1 / 10$ & $1 / 10$ & $1 / 10$ & $1 / 10$ & $1 / 10$ \\
\hline & & 1000 & Normal & $15 / 15$ & $14 / 15$ & $14 / 15$ & $14 / 15$ & $14 / 15$ & $14 / 15$ & $14 / 15$ & $12 / 15$ & $12 / 15$ & $12 / 15$ \\
\hline & & & Edema & $0 / 15$ & $1 / 15$ & $1 / 15$ & $1 / 15$ & $1 / 15$ & $1 / 15$ & $1 / 15$ & $3 / 15$ & $3 / 15$ & $3 / 15$ \\
\hline
\end{tabular}

Data represented number of animal which clinical sign was observed/total animal number in the group.

criterion. Toxicokinetic analysis was performed by Phoe$\mathrm{nix}^{\mathrm{TM}}$ WinNonlin $^{\circledR}$ (ver. 6.2, Pharsight) using the mean serum-concentrations at each time. Analysis model used the non-compartment model, and the area under the curve $\left(\mathrm{AUC}_{0-24 \mathrm{~h}}\right)$ and $\mathrm{C}_{0}$ were determined.

Statistical analysis. The collected data for the toxicity test were statistically analyzed with the commercial program SPSS 10.1, and the significance level was set at $p<$ 0.05 . Body weight, food and water consumption, total volume of urine, hematological and clinical biochemistry tests, and organ weight were assumed to be normally distributed and analyzed by one-way ANOVA. The Student's $t$-test was used to test for a difference between the means of two independent groups (G1 vs. G2, G2 vs. G5). The rank transformation was performed for the urinalysis data and analyzed by the non-parametric Kruskal-Wallis' H-test.

Histopathological examination data was analyzed by the non-parametric Fisher's exact 2-tailed test using the statistical package included within Pristima. In this report, 'significant' means that it was statistically significant compared with vehicle control group unless otherwise noted.

For the TK test, the mean, standard deviation, relative error, and coefficient of variation were calculated as below equations using Microsoft ${ }^{\mathbb{}}$ Office Excel $^{\circledR} 2007$.

\section{RESULTS}

Clinical signs. No mortality was observed in any group. In the TS-DP2 groups, edema of one or both hind limbs was observed in 1 6 cases on Day 10 29 in males, and 1 or 3 cases on Day 21 30 in females, at the dose of $1000 \mu \mathrm{g} / \mathrm{kg} /$
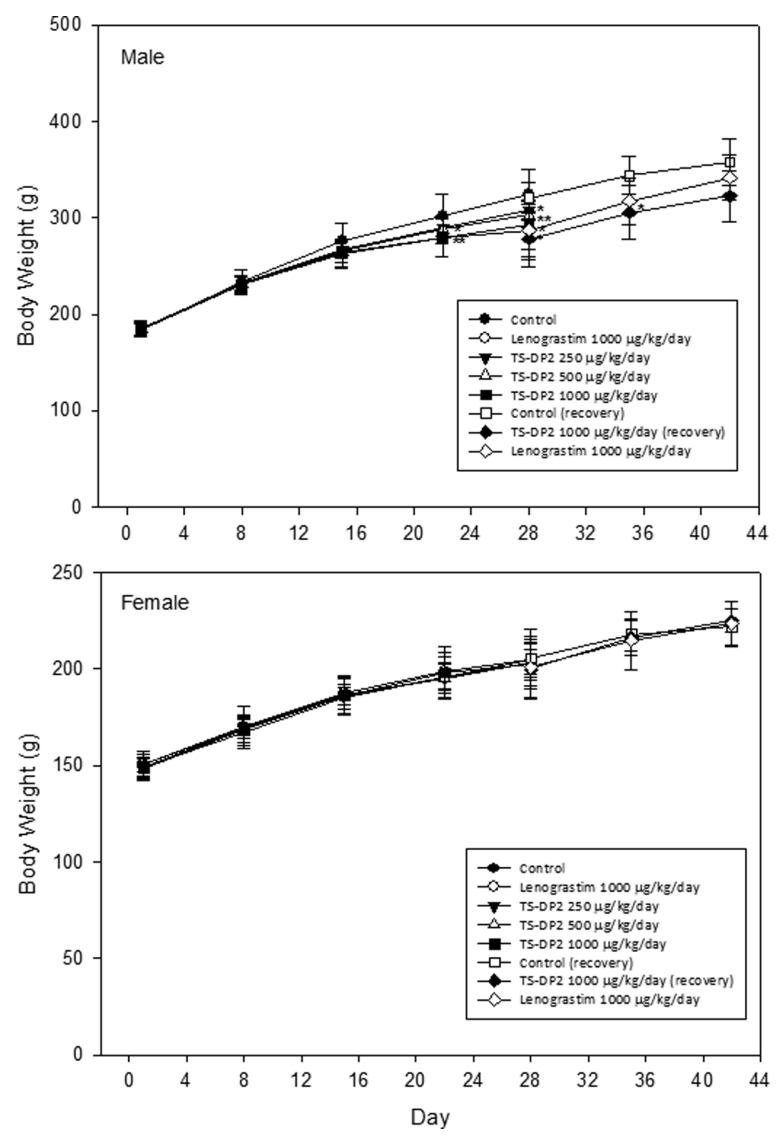

Fig. 1. Change of body weights in male and female SpragueDawley rats intravenously treated with TS-DP2 for 4 weeks. The day of first treatment was designated Day 1. Each value represents mean \pm SD $(n=10$ or 15$)$. ***: Significant difference at $p<0.05 / p<0.01$ levels compared with the negative control. 

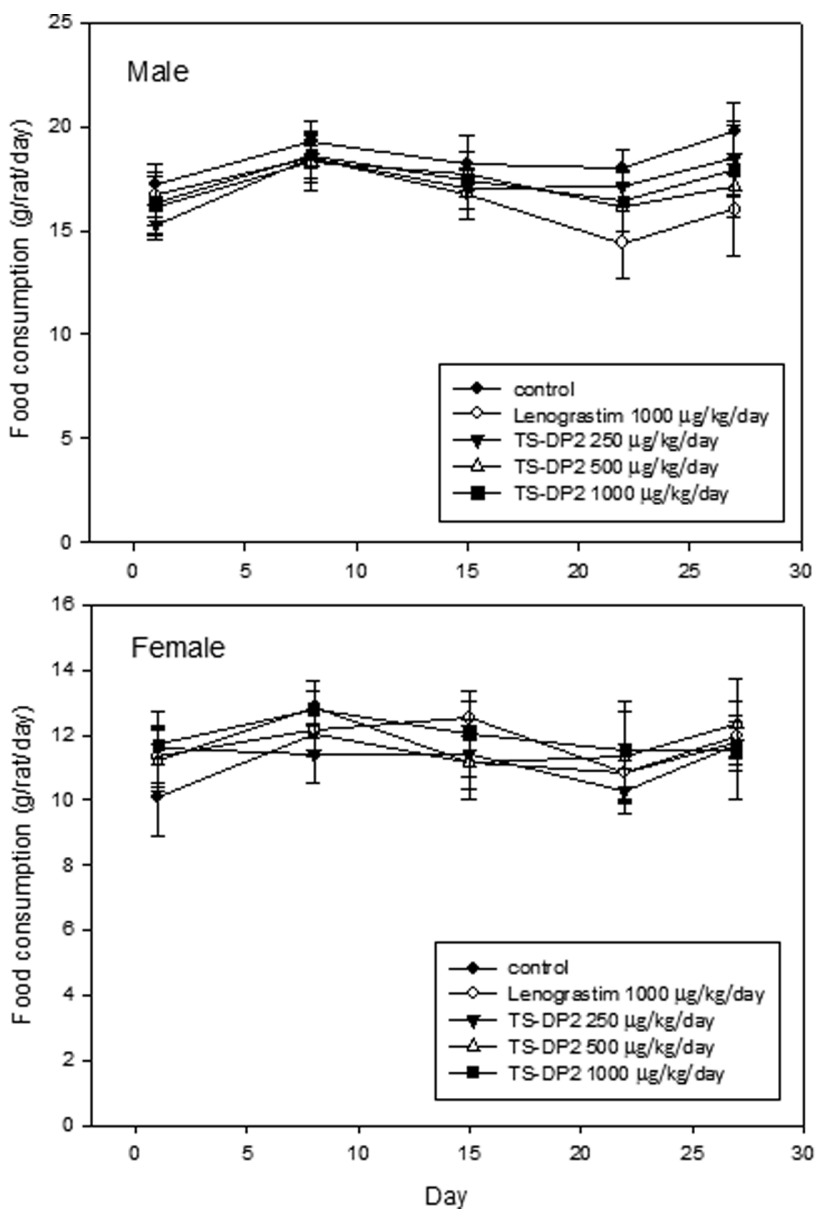

Fig. 2. Food consumption in male and female Sprague-Dawley rats intravenously treated with TS-DP2 for 4 weeks. No significant changes were observed in all TS-DP2 administration groups as compared with control. Each value represents mean $\pm S D(n=10$ or 15$)$.

day (G5), and in 1 case on Day 21 30 in females at the dose of $500 \mu \mathrm{g} / \mathrm{kg} /$ day (G4) (Table 2). In addition, abnormal gait or ataxia was observed in 1 case on Day 10 35 in males at the dose of $1000 \mu \mathrm{g} / \mathrm{kg} /$ day (G5). In the lenograstim group (G2), edema of one or both hind limbs was observed in 2 7 cases on Day 22 29 in males and in 1 2 cases on Day 22 30 in females. Edema of one or both hind limbs was observed in 1 3 cases on Day 30 43 (recovery period) in males and in 1 case on Day 31 45 in females at the dose of $1000 \mu \mathrm{g} / \mathrm{kg} / \mathrm{day}$, which represents a gradual decrease over time. Ataxia was observed in 1 case (male) at the dose of $1000 \mu \mathrm{g} / \mathrm{kg} /$ day on Day 30 35 and then recovered. In the lenograstim group (G2), edema of one or both hind limbs was observed in 2 cases on Day 30 35 in males and in 1 case on Day 31 43 in females.

Body weight changes, food and water consumption. The mean body weight on Days 22 and 28 and the body
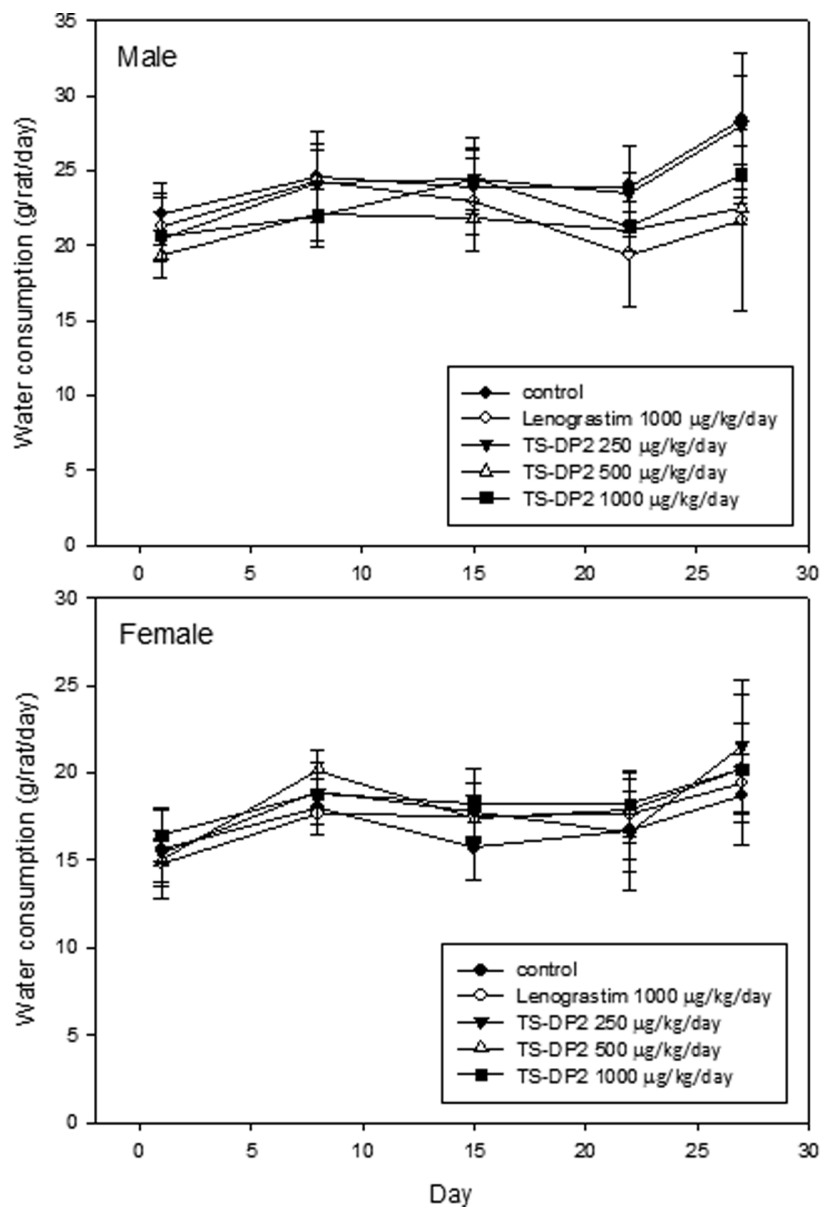

Fig. 3. Water consumption in male and female Sprague-Dawley rats intravenously treated with TS-DP2 for 4 weeks. No significant changes were observed in all TS-DP2 administration groups as compared with control. Each value represents mean $\pm S D(n=10$ or 15$)$.

weight gain at the highest dose of either TS-DP2 and lenograstim in males were significantly lower than the negative control $(p<0.05$ or $p<0.01)$ (Fig. 1). During the recovery period, the mean body weight on Day 28 for TS-DP2 and on Day 28 and 35 for lenograstim in males was significantly lower than negative control $(p<0.05)$. Food consumption and water consumption were similar between the two groups (Fig. 2 and 3). No abnormal findings or toxicologically significant differences were observed in all groups on ophthalmological examination and urinalysis (data not shown).

Hematological test. Statistical significance compared to negative control (G1) in hematological test is shown in Table 3 and 4. MCV (except females at the dose of $250 \mu \mathrm{g} /$ $\mathrm{kg}$ /day), RDW, the number and percentage of WBC and NEU, LYM, MONO, EOS (except males at the dose of $1000 \mu \mathrm{g} / \mathrm{kg} /$ day), BASO, and LUC of both sexes were significantly higher at all doses $(p<0.01)$. LUC of males at all 
Table 3. Hematological findings of SD rats intravenously treated with TS-DP2 for 4 weeks

\begin{tabular}{|c|c|c|c|c|c|c|}
\hline \multirow{2}{*}{ Item } & \multirow{2}{*}{ Units } & \multicolumn{5}{|c|}{ Group $(\mu \mathrm{g} / \mathrm{kg} /$ day) } \\
\hline & & G1 (0) & G2 (1000) & G3 (250) & G4 (500) & G5 (1000) \\
\hline \multicolumn{7}{|l|}{ Male } \\
\hline $\mathrm{RBC}$ & $10^{6} / \mu \mathrm{L}$ & $7.91 \pm 0.23$ & $7.22 \pm 0.20^{* * *}$ & $7.37 \pm 0.34^{* *}$ & $7.63 \pm 0.24^{*}$ & $7.38 \pm 0.28^{* * *}$ \\
\hline HGB & $\mathrm{g} / \mathrm{dL}$ & $14.9 \pm 0.5$ & $14.3 \pm 0.4^{* * *}$ & $14.4 \pm 0.4$ & $14.7 \pm 0.4$ & $14.5 \pm 0.7$ \\
\hline HCT & $\%$ & $45.1 \pm 1.4$ & $43.2 \pm 1.2^{* *}$ & $43.1 \pm 1.2^{*}$ & $44.7 \pm 1.6$ & $43.7 \pm 2.1$ \\
\hline $\mathrm{MCV}$ & $\mathrm{fL}$ & $57.0 \pm 1.0$ & $59.8 \pm 1.8^{* *}$ & $58.6 \pm 1.6^{*}$ & $58.6 \pm 1.6^{*}$ & $59.2 \pm 1.9^{* * *}$ \\
\hline $\mathrm{MCH}$ & pg & $18.9 \pm 0.5$ & $19.9 \pm 0.6^{* *}$ & $19.5 \pm 0.6^{*}$ & $19.3 \pm 0.5$ & $19.6 \pm 0.6^{*}$ \\
\hline $\mathrm{MCHC}$ & $\mathrm{g} / \mathrm{dL}$ & $33.1 \pm 0.7$ & $33.2 \pm 0.5$ & $33.3 \pm 0.2$ & $33.0 \pm 0.5$ & $33.0 \pm 0.3$ \\
\hline RDW & $\%$ & $11.6 \pm 0.4$ & $12.4 \pm 0.2^{* *}$ & $11.9 \pm 0.4^{*}$ & $12.2 \pm 0.2^{* *}$ & $12.6 \pm 0.3^{* *}$ \\
\hline RET & $\%$ & $3.78 \pm 0.26$ & $3.49 \pm 0.81$ & $3.38 \pm 0.53$ & $3.52 \pm 0.33$ & $3.67 \pm 0.74$ \\
\hline PLT & $10^{3} / \mu \mathrm{L}$ & $999.0 \pm 47.5$ & $786.9 \pm 120.9^{* *}$ & $917.3 \pm 110.6^{*}$ & $797.9 \pm 81.4^{* * *}$ & $756.5 \pm 89.9^{* * *}$ \\
\hline MPV & $\mathrm{fL}$ & $5.19 \pm 0.25$ & $6.11 \pm 0.45^{* *}$ & $5.55 \pm 0.50^{*}$ & $5.84 \pm 0.30^{* *}$ & $6.10 \pm 0.39^{* * *}$ \\
\hline WBC & $10^{3} / \mu \mathrm{L}$ & $9.66 \pm 0.99$ & $81.62 \pm 13.63^{* *}$ & $38.84 \pm 7.09^{* *}$ & $59.71 \pm 7.06^{* *}$ & $77.87 \pm 9.38^{* * *}$ \\
\hline NEU & $\%$ & $12.1 \pm 2.8$ & $79.4 \pm 2.7^{* *}$ & $66.4 \pm 4.1^{* *}$ & $74.4 \pm 3.3^{* * *}$ & $78.7 \pm 4.2^{* *}$ \\
\hline LYM & $\%$ & $82.6 \pm 3.2$ & $16.6 \pm 2.3^{* *}$ & $28.6 \pm 3.3^{* *}$ & $21.4 \pm 3.1^{* *}$ & $17.0 \pm 3.0^{* *}$ \\
\hline MONO & $\%$ & $3.45 \pm 0.83$ & $1.99 \pm 0.87^{* *}$ & $2.70 \pm 0.60^{*}$ & $2.54 \pm 0.45^{*}$ & $2.16 \pm 1.13^{* *}$ \\
\hline EOS & $\%$ & $0.66 \pm 0.18$ & $0.26 \pm 0.15^{* *}$ & $0.54 \pm 0.21$ & $0.31 \pm 0.06^{* *}$ & $0.31 \pm 0.26^{*}$ \\
\hline BASO & $\%$ & $0.17 \pm 0.07$ & $0.33 \pm 0.08^{* *}$ & $0.22 \pm 0.04$ & $0.29 \pm 0.03^{* *}$ & $0.32 \pm 0.06^{* * *}$ \\
\hline LUC & $\%$ & $1.04 \pm 0.20$ & $1.46 \pm 0.24^{\text {*** }}$ & $1.58 \pm 0.58^{* *}$ & $1.15 \pm 0.22$ & $1.57 \pm 0.30^{* * *}$ \\
\hline PT & $\mathrm{sec}$ & $8.8 \pm 0.5$ & $9.0 \pm 0.4$ & $9.1 \pm 0.5$ & $9.1 \pm 0.3$ & $9.1 \pm 0.3$ \\
\hline APTT & $\mathrm{sec}$ & $18.2 \pm 0.7$ & $18.1 \pm 0.8$ & $18.3 \pm 0.5$ & $18.9 \pm 0.5^{*}$ & $18.8 \pm 0.6^{*}$ \\
\hline \multicolumn{7}{|l|}{ Female } \\
\hline $\mathrm{RBC}$ & $10^{6} / \mu \mathrm{L}$ & $7.25 \pm 0.14$ & $6.82 \pm 0.24^{* * *}$ & $7.15 \pm 0.16$ & $6.96 \pm 0.25^{*}$ & $6.99 \pm 0.35$ \\
\hline HGB & $\mathrm{g} / \mathrm{dL}$ & $13.5 \pm 0.3$ & $13.2 \pm 0.4$ & $13.5 \pm 0.4$ & $13.3 \pm 0.5$ & $13.2 \pm 0.5$ \\
\hline HCT & $\%$ & $40.3 \pm 1.1$ & $40.1 \pm 1.0$ & $40.5 \pm 0.9$ & $40.3 \pm 1.7$ & $40.0 \pm 1.8$ \\
\hline $\mathrm{MCV}$ & $\mathrm{fL}$ & $55.5 \pm 1.0$ & $58.8 \pm 2.0^{* * *}$ & $56.6 \pm 1.8$ & $57.8 \pm 1.2^{* * *}$ & $57.2 \pm 2.3^{*}$ \\
\hline $\mathrm{MCH}$ & pg & $18.6 \pm 0.4$ & $19.4 \pm 0.8^{*}$ & $18.8 \pm 0.6$ & $19.2 \pm 0.3$ & $18.8 \pm 0.8$ \\
\hline $\mathrm{MCHC}$ & $\mathrm{g} / \mathrm{dL}$ & $33.6 \pm 0.5$ & $33.1 \pm 0.3^{*}$ & $33.3 \pm 0.7$ & $33.1 \pm 0.3$ & $32.9 \pm 0.5$ \\
\hline RDW & $\%$ & $11.1 \pm 0.3$ & $12.3 \pm 0.5^{* *}$ & $12.0 \pm 0.5^{* * *}$ & $12.4 \pm 0.3^{* * *}$ & $12.5 \pm 0.4^{* * *}$ \\
\hline RET & $\%$ & $3.52 \pm 0.70$ & $4.66 \pm 0.63^{* *}$ & $3.67 \pm 0.92$ & $4.50 \pm 0.84^{*}$ & $4.53 \pm 1.10^{*}$ \\
\hline PLT & $10^{3} / \mu \mathrm{L}$ & $1020.8 \pm 59.8$ & $909.4 \pm 110.8^{*}$ & $1024.6 \pm 136.6$ & $978.1 \pm 191.8$ & $962.4 \pm 196.2$ \\
\hline MPV & $\mathrm{fL}$ & $5.76 \pm 0.70$ & $6.56 \pm 0.71^{*}$ & $6.09 \pm 0.63$ & $6.24 \pm 0.70$ & $6.35 \pm 0.64$ \\
\hline WBC & $10^{3} / \mu \mathrm{L}$ & $5.50 \pm 1.04$ & $42.36 \pm 6.76^{* * *}$ & $19.08 \pm 4.09^{* * *}$ & $25.97 \pm 5.24^{* *}$ & $35.95 \pm 7.85^{* * *}$ \\
\hline NEU & $\%$ & $10.6 \pm 3.3$ & $73.1 \pm 2.4^{* * *}$ & $60.4 \pm 5.6^{* *}$ & $65.6 \pm 3.3^{* *}$ & $70.3 \pm 5.0^{* * *}$ \\
\hline LYM & $\%$ & $84.4 \pm 3.4$ & $23.3 \pm 2.1^{* *}$ & $35.4 \pm 4.8^{* *}$ & $30.5 \pm 3.0^{* * *}$ & $26.2 \pm 4.9^{* * *}$ \\
\hline MONO & $\%$ & $2.70 \pm 0.48$ & $1.72 \pm 0.50^{* * *}$ & $2.12 \pm 0.60^{* *}$ & $1.76 \pm 0.45^{* *}$ & $1.56 \pm 0.35^{* *}$ \\
\hline EOS & $\%$ & $1.27 \pm 0.18$ & $0.68 \pm 0.28^{* *}$ & $0.89 \pm 0.29^{* *}$ & $0.89 \pm 0.26^{* *}$ & $0.69 \pm 0.29^{* *}$ \\
\hline BASO & $\%$ & $0.15 \pm 0.08$ & $0.19 \pm 0.06$ & $0.14 \pm 0.05$ & $0.16 \pm 0.05$ & $0.21 \pm 0.06$ \\
\hline LUC & $\%$ & $0.90 \pm 0.19$ & $0.98 \pm 0.23$ & $1.09 \pm 0.37$ & $1.12 \pm 0.51$ & $1.02 \pm 0.21$ \\
\hline PT & $\mathrm{sec}$ & $8.7 \pm 0.4$ & $8.7 \pm 0.3$ & $8.9 \pm 0.3$ & $8.7 \pm 0.2$ & $8.9 \pm 0.3$ \\
\hline APTT & $\mathrm{sec}$ & $17.8 \pm 1.7$ & $17.7 \pm 0.7$ & $17.9 \pm 0.8$ & $17.8 \pm 0.7$ & $17.9 \pm 0.7$ \\
\hline
\end{tabular}

Data are expressed as Mean \pm SD $(\mathrm{n}=10)$. Statistically significant from G1 $\left({ }^{*} p<0.05 ;{ }^{* *} p<0.01\right)$ or G2 $\left({ }^{*} p<0.05\right)$. G1: Negative control, G2: Reference control, G3-G5: Test article treatment groups.

$\mathrm{RBC}$, red blood cell; $\mathrm{HGB}$, hemoglobin; $\mathrm{HCT}$, hematocrit; $\mathrm{MCV}$, mean corpuscular volume; $\mathrm{MCH}$, mean corpuscularhemoglobin; MCHC, mean corpuscular hemoglobin concentration; RDW, red cell distribution width; RET, reticulocytes; PLT, platelet; WBC, white blood cell; NEU, neutrophil; LYM, lymphocyte; MONO, monocyte; EOS, eosinophil; BASO, basophil; LUC, large unstained cell; PT, prothrombin time; APTT, activated partial thromboplastin time.

doses were significantly increased $(p<0.05$ or $p<0.01)$ and percentage of RET of females at the dose of $500 \mu \mathrm{g} / \mathrm{kg} /$ day or less was significantly increased $(p<0.05$ or $p<$ $0.01)$. RBC and PLT of males at all doses were significantly decreased $(p<0.01)$. RBC in females at the dose of $500 \mu \mathrm{g} / \mathrm{kg} /$ day was significantly decreased $(p<0.05)$. The percentage of LYM, MONO, and EOS (except in males at the dose of $250 \mu \mathrm{g} / \mathrm{kg} /$ day) of both sexes was significantly decreased $(p<0.01)$, but it was considered to be relative changes according to the change on WBC number. Although APTT of males at the dose of $500 \mu \mathrm{g} / \mathrm{kg} /$ day or less was significantly decreased $(p<0.05)$, it was within normal ref- 
Table 4. Hematological findings in 2-week recovery groups with TS-DP2 after 4-week treatment

\begin{tabular}{|c|c|c|c|c|}
\hline \multirow{2}{*}{ Item } & \multirow{2}{*}{ Units } & \multicolumn{3}{|c|}{ Group ( $\mu \mathrm{g} / \mathrm{kg} /$ day) } \\
\hline & & G1 (0) & G2 (1000) & G5 (1000) \\
\hline \multicolumn{5}{|l|}{ Male } \\
\hline $\mathrm{RBC}$ & $10^{6} / \mu \mathrm{L}$ & $8.32 \pm 0.18$ & $8.39 \pm 0.32$ & $8.09 \pm 0.42$ \\
\hline HGB & $\mathrm{g} / \mathrm{dL}$ & $14.9 \pm 0.3$ & $15.2 \pm 0.5$ & $14.8 \pm 0.5$ \\
\hline HCT & $\%$ & $45.5 \pm 0.8$ & $46.6 \pm 2.0$ & $45.3 \pm 1.7$ \\
\hline $\mathrm{MCV}$ & $\mathrm{fL}$ & $54.7 \pm 0.9$ & $55.6 \pm 3.2$ & $56.0 \pm 1.1$ \\
\hline $\mathrm{MCH}$ & pg & $17.9 \pm 0.3$ & $18.2 \pm 1.0$ & $18.4 \pm 0.4$ \\
\hline $\mathrm{MCHC}$ & $\mathrm{g} / \mathrm{dL}$ & $32.8 \pm 0.3$ & $32.8 \pm 0.3$ & $32.8 \pm 0.6$ \\
\hline RDW & $\%$ & $11.2 \pm 0.2$ & $12.0 \pm 0.2^{* *}$ & $12.2 \pm 1.0$ \\
\hline RET & $\%$ & $2.61 \pm 0.41$ & $3.27 \pm 0.47^{*}$ & $4.09 \pm 1.24^{*}$ \\
\hline PLT & $10^{3} / \mu \mathrm{L}$ & $871.2 \pm 63.0$ & $878.8 \pm 68.2$ & $890.6 \pm 158.1$ \\
\hline MPV & $\mathrm{fL}$ & $5.06 \pm 0.15$ & $5.08 \pm 0.08$ & $5.30 \pm 0.25$ \\
\hline WBC & $10^{3} / \mu \mathrm{L}$ & $8.94 \pm 1.15$ & $7.29 \pm 0.99^{*}$ & $8.90 \pm 0.99^{\#}$ \\
\hline NEU & $\%$ & $15.0 \pm 6.7$ & $13.4 \pm 3.6$ & $11.8 \pm 3.6$ \\
\hline LYM & $\%$ & $78.7 \pm 7.7$ & $80.3 \pm 3.7$ & $81.8 \pm 3.7$ \\
\hline MONO & $\%$ & $4.30 \pm 1.01$ & $3.78 \pm 1.08$ & $4.12 \pm 0.97$ \\
\hline EOS & $\%$ & $0.76 \pm 0.19$ & $1.02 \pm 0.19$ & $0.94 \pm 0.15$ \\
\hline BASO & $\%$ & $0.16 \pm 0.05$ & $0.14 \pm 0.05$ & $0.18 \pm 0.08$ \\
\hline LUC & $\%$ & $1.00 \pm 0.24$ & $1.38 \pm 0.36$ & $1.10 \pm 0.35$ \\
\hline PT & $\mathrm{sec}$ & $9.5 \pm 0.3$ & $9.9 \pm 0.4$ & $10.0 \pm 0.3^{*}$ \\
\hline APTT & $\mathrm{sec}$ & $18.4 \pm 0.6$ & $18.5 \pm 0.5$ & $18.2 \pm 0.4$ \\
\hline \multicolumn{5}{|l|}{ Female } \\
\hline $\mathrm{RBC}$ & $10^{6} / \mu \mathrm{L}$ & $7.68 \pm 0.22$ & $7.62 \pm 0.18$ & $7.62 \pm 0.30$ \\
\hline HGB & $\mathrm{g} / \mathrm{dL}$ & $14.0 \pm 0.3$ & $14.2 \pm 0.5$ & $14.1 \pm 0.5$ \\
\hline $\mathrm{HCT}$ & $\%$ & $41.7 \pm 0.5$ & $42.8 \pm 1.2$ & $42.4 \pm 1.4$ \\
\hline $\mathrm{MCV}$ & $\mathrm{fL}$ & $54.3 \pm 1.3$ & $56.2 \pm 2.1$ & $55.7 \pm 1.3$ \\
\hline $\mathrm{MCH}$ & pg & $18.3 \pm 0.4$ & $18.7 \pm 0.9$ & $18.5 \pm 0.4$ \\
\hline MCHC & $\mathrm{g} / \mathrm{dL}$ & $33.7 \pm 0.5$ & $33.2 \pm 0.4$ & $33.2 \pm 0.4$ \\
\hline RDW & $\%$ & $10.9 \pm 0.4$ & $11.6 \pm 0.3^{*}$ & $11.4 \pm 0.3^{*}$ \\
\hline RET & $\%$ & $2.28 \pm 0.56$ & $3.44 \pm 0.64^{*}$ & $2.96 \pm 0.71$ \\
\hline PLT & $10^{3} / \mu \mathrm{L}$ & $1006.6 \pm 111.3$ & $967.6 \pm 101.5$ & $986.0 \pm 80.1$ \\
\hline MPV & $\mathrm{fL}$ & $4.86 \pm 0.15$ & $4.88 \pm 0.26$ & $4.82 \pm 0.11$ \\
\hline WBC & $10^{3} / \mu \mathrm{L}$ & $4.51 \pm 0.52$ & $5.85 \pm 0.63^{* * *}$ & $4.96 \pm 0.93$ \\
\hline NEU & $\%$ & $12.0 \pm 1.8$ & $13.5 \pm 2.1$ & $11.5 \pm 3.5$ \\
\hline LYM & $\%$ & $82.1 \pm 2.5$ & $79.9 \pm 2.7$ & $82.1 \pm 3.7$ \\
\hline MONO & $\%$ & $2.88 \pm 0.99$ & $3.38 \pm 0.80$ & $2.66 \pm 0.79$ \\
\hline EOS & $\%$ & $1.74 \pm 0.67$ & $2.14 \pm 0.69$ & $2.48 \pm 1.41$ \\
\hline BASO & $\%$ & $0.10 \pm 0.00$ & $0.14 \pm 0.09$ & $0.12 \pm 0.08$ \\
\hline LUC & $\%$ & $1.14 \pm 0.15$ & $0.98 \pm 0.16$ & $1.14 \pm 0.23$ \\
\hline PT & sec & $9.2 \pm 0.3$ & $9.8 \pm 0.3^{*}$ & $9.7 \pm 0.6$ \\
\hline APTT & $\mathrm{sec}$ & $16.6 \pm 1.5$ & $17.5 \pm 0.5$ & $16.9 \pm 0.3^{\#}$ \\
\hline
\end{tabular}

Data are expressed as Mean \pm SD $(n=5)$. Statistically significant from G1 ( $\left.{ }^{*} p<0.05 ;{ }^{* *} p<0.01\right)$ or G2 ( $\left.{ }^{*} p<0.05\right)$. G1: Negative control, G2: Reference control, G3-G5: Test article treatment groups.

$\mathrm{RBC}$, red blood cell; HGB, hemoglobin; $\mathrm{HCT}$, hematocrit; MCV, mean corpuscular volume; $\mathrm{MCH}$, mean corpuscularhemoglobin; MCHC, mean corpuscular hemoglobin concentration; RDW, red cell distribution width; RET, reticulocytes; PLT, platelet; WBC, white blood cell; NEU, neutrophil; LYM, lymphocyte; MONO, monocyte; EOS, eosinophil; BASO, basophil; LUC, large unstained cell; PT, prothrombin time; APTT, activated partial thromboplastin time.

erence range provided by Chemon, Inc. Other changes were considered to be unrelated to TS-DP2, because these changes were observed without dose-response relationship. In the lenograstim group, MCV, MCH, RDW, MPV, the number and percentage of WBC and NEU, LYM, MONO, EOS, $\mathrm{BASO}$, and LUC of both sexes were significantly increased $(p<0.05$ or $p<0.01)$. The percentage of BASO and LUC of males and the percentage of RET of females were significantly increased $(p<0.05$ or $p<0.01)$. RBC and PLT of both sexes were significantly decreased $(p<0.05$ or $p<$ $0.01)$. HGB and the percentage of HCT of males, and MCHC of females were significantly decreased $(p<0.05$ or $p<$ 
Table 5. Serum biochemical findings of SD rats intravenously treated with TS-DP2 for 4 weeks

\begin{tabular}{|c|c|c|c|c|c|c|}
\hline \multirow{2}{*}{ Item } & \multirow{2}{*}{ Units } & \multicolumn{5}{|c|}{ Group ( $\mu \mathrm{g} / \mathrm{kg} /$ day) } \\
\hline & & G1 (0) & G2 (1000) & G3 (250) & G4 (500) & G5 (1000) \\
\hline \multicolumn{7}{|l|}{ Male } \\
\hline AST & $\mathrm{U} / \mathrm{L}$ & $78.4 \pm 11.0$ & $103.2 \pm 20.1^{* * *}$ & $88.5 \pm 7.2^{*}$ & $88.7 \pm 5.6^{*}$ & $94.9 \pm 12.9^{* * *}$ \\
\hline ALT & $\mathrm{U} / \mathrm{L}$ & $35.8 \pm 4.8$ & $38.3 \pm 6.3$ & $36.6 \pm 3.0$ & $36.7 \pm 5.2$ & $36.1 \pm 6.9$ \\
\hline ALP & $\mathrm{U} / \mathrm{L}$ & $125.5 \pm 16.7$ & $1265.0 \pm 175.8^{* * *}$ & $725.5 \pm 202.9^{* *}$ & $1143.3 \pm 185.2^{* *}$ & $1461.5 \pm 218.6^{\ddagger * *}$ \\
\hline CPK & $\mathrm{U} / \mathrm{L}$ & $160.5 \pm 48.8$ & $137.9 \pm 30.0$ & $138.8 \pm 49.9$ & $125.0 \pm 40.0$ & $126.9 \pm 22.1$ \\
\hline TBIL & $\mathrm{mg} / \mathrm{dL}$ & $0.14 \pm 0.01$ & $0.14 \pm 0.01$ & $0.14 \pm 0.01$ & $0.14 \pm 0.01$ & $0.14 \pm 0.01$ \\
\hline GLU & $\mathrm{mg} / \mathrm{dL}$ & $115.6 \pm 12.2$ & $119.8 \pm 12.2$ & $113.2 \pm 13.7$ & $129.3 \pm 18.3$ & $121.3 \pm 12.9$ \\
\hline TCHO & $\mathrm{mg} / \mathrm{dL}$ & $91.7 \pm 15.8$ & $78.1 \pm 19.0$ & $78.4 \pm 9.6^{*}$ & $67.9 \pm 16.5^{* *}$ & $73.3 \pm 7.3^{* *}$ \\
\hline TG & $\mathrm{mg} / \mathrm{dL}$ & $42.3 \pm 9.3$ & $45.9 \pm 22.2$ & $43.4 \pm 12.3$ & $47.2 \pm 10.6$ & $48.3 \pm 12.6$ \\
\hline $\mathrm{TP}$ & $\mathrm{g} / \mathrm{dL}$ & $5.72 \pm 0.21$ & $5.47 \pm 0.21^{*}$ & $5.65 \pm 0.16$ & $5.68 \pm 0.15$ & $5.49 \pm 0.24$ \\
\hline ALB & $\mathrm{g} / \mathrm{dL}$ & $3.03 \pm 0.12$ & $2.91 \pm 0.20$ & $3.03 \pm 0.15$ & $3.08 \pm 0.11$ & $2.99 \pm 0.18$ \\
\hline A/Gratio & & $1.13 \pm 0.05$ & $1.14 \pm 0.08$ & $1.16 \pm 0.09$ & $1.19 \pm 0.10$ & $1.19 \pm 0.09$ \\
\hline BUN & $\mathrm{mg} / \mathrm{dL}$ & $18.0 \pm 1.2$ & $17.6 \pm 1.3$ & $17.0 \pm 1.6$ & $18.5 \pm 2.7$ & $18.3 \pm 2.0$ \\
\hline CRE & $\mathrm{mg} / \mathrm{dL}$ & $0.41 \pm 0.02$ & $0.41 \pm 0.04$ & $0.43 \pm 0.04$ & $0.43 \pm 0.03$ & $0.40 \pm 0.03$ \\
\hline IP & $\mathrm{mg} / \mathrm{dL}$ & $8.08 \pm 0.44$ & $7.88 \pm 0.23$ & $8.18 \pm 0.30$ & $7.74 \pm 0.35$ & $8.03 \pm 0.37$ \\
\hline $\mathrm{Ca}^{2+}$ & $\mathrm{mg} / \mathrm{dL}$ & $10.06 \pm 0.32$ & $9.97 \pm 0.27$ & $10.00 \pm 0.29$ & $10.02 \pm 0.18$ & $9.85 \pm 0.25$ \\
\hline $\mathrm{Na}^{+}$ & $\mathrm{mmol} / \mathrm{L}$ & $141.0 \pm 0.7$ & $141.9 \pm 0.6^{* * *}$ & $141.2 \pm 0.8$ & $141.4 \pm 0.8$ & $141.7 \pm 0.8$ \\
\hline $\mathrm{K}^{+}$ & $\mathrm{mmol} / \mathrm{L}$ & $4.96 \pm 0.17$ & $4.64 \pm 0.26^{* *}$ & $4.65 \pm 0.16^{* *}$ & $4.65 \pm 0.16^{* * *}$ & $4.50 \pm 0.20^{* *}$ \\
\hline $\mathrm{Cl}^{-}$ & $\mathrm{mmol} / \mathrm{L}$ & $102.7 \pm 0.9$ & $104.4 \pm 1.3^{* *}$ & $103.7 \pm 1.3^{*}$ & $104.0 \pm 1.0^{* * *}$ & $104.3 \pm 0.9^{* *}$ \\
\hline \multicolumn{7}{|l|}{ Female } \\
\hline AST & $\mathrm{U} / \mathrm{L}$ & $71.3 \pm 5.8$ & $84.5 \pm 7.2^{* *}$ & $77.0 \pm 7.8$ & $75.0 \pm 7.5$ & $82.2 \pm 8.1^{* *}$ \\
\hline ALT & $\mathrm{U} / \mathrm{L}$ & $28.0 \pm 4.4$ & $25.7 \pm 2.3$ & $28.0 \pm 3.3$ & $26.2 \pm 1.9$ & $27.0 \pm 3.8$ \\
\hline ALP & $\mathrm{U} / \mathrm{L}$ & $68.5 \pm 9.0$ & $1069.4 \pm 178.1^{* * *}$ & $561.2 \pm 124.6^{* *}$ & $739.2 \pm 127.8^{* *}$ & $1036.8 \pm 118.1^{* * *}$ \\
\hline $\mathrm{CPK}$ & $\mathrm{U} / \mathrm{L}$ & $132.2 \pm 43.8$ & $95.9 \pm 23.7^{*}$ & $117.8 \pm 48.4$ & $95.2 \pm 23.8$ & $119.6 \pm 39.7$ \\
\hline TBIL & $\mathrm{mg} / \mathrm{dL}$ & $0.15 \pm 0.01$ & $0.15 \pm 0.01$ & $0.15 \pm 0.01$ & $0.15 \pm 0.01$ & $0.14 \pm 0.01$ \\
\hline GLU & $\mathrm{mg} / \mathrm{dL}$ & $115.3 \pm 9.1$ & $122.3 \pm 11.5$ & $121.2 \pm 8.0$ & $126.4 \pm 9.3$ & $117.9 \pm 9.5$ \\
\hline TCHO & $\mathrm{mg} / \mathrm{dL}$ & $87.7 \pm 13.0$ & $87.2 \pm 16.2$ & $81.9 \pm 19.9$ & $95.8 \pm 19.5$ & $80.4 \pm 17.3$ \\
\hline $\mathrm{TG}$ & $\mathrm{mg} / \mathrm{dL}$ & $34.3 \pm 9.7$ & $32.9 \pm 10.1$ & $30.6 \pm 6.0$ & $33.2 \pm 8.3$ & $36.1 \pm 9.3$ \\
\hline $\mathrm{TP}$ & $\mathrm{g} / \mathrm{dL}$ & $5.52 \pm 0.14$ & $5.43 \pm 0.23$ & $5.51 \pm 0.26$ & $5.51 \pm 0.14$ & $5.41 \pm 0.28$ \\
\hline ALB & $\mathrm{g} / \mathrm{dL}$ & $3.09 \pm 0.06$ & $3.08 \pm 0.19$ & $3.13 \pm 0.16$ & $3.07 \pm 0.19$ & $3.00 \pm 0.22$ \\
\hline A/Gratio & & $1.27 \pm 0.04$ & $1.32 \pm 0.09$ & $1.32 \pm 0.08$ & $1.26 \pm 0.15$ & $1.25 \pm 0.15$ \\
\hline BUN & $\mathrm{mg} / \mathrm{dL}$ & $19.5 \pm 1.9$ & $21.5 \pm 2.4$ & $21.1 \pm 1.9$ & $21.1 \pm 2.5$ & $20.0 \pm 1.8$ \\
\hline CRE & $\mathrm{mg} / \mathrm{dL}$ & $0.45 \pm 0.03$ & $0.50 \pm 0.05^{\text {*** }}$ & $0.48 \pm 0.02$ & $0.46 \pm 0.03$ & $0.45 \pm 0.03^{\# \#}$ \\
\hline IP & $\mathrm{mg} / \mathrm{dL}$ & $7.26 \pm 0.73$ & $7.55 \pm 0.69$ & $7.70 \pm 0.55$ & $7.44 \pm 0.52$ & $7.71 \pm 0.80$ \\
\hline $\mathrm{Ca}^{2+}$ & $\mathrm{mg} / \mathrm{dL}$ & $9.77 \pm 0.19$ & $9.83 \pm 0.24$ & $9.86 \pm 0.27$ & $9.87 \pm 0.13$ & $9.84 \pm 0.26$ \\
\hline $\mathrm{Na}^{+}$ & $\mathrm{mmol} / \mathrm{L}$ & $135.4 \pm 1.1$ & $136.5 \pm 1.2$ & $136.2 \pm 1.2$ & $136.6 \pm 1.1$ & $135.9 \pm 1.0$ \\
\hline $\mathrm{K}^{+}$ & $\mathrm{mmol} / \mathrm{L}$ & $4.87 \pm 0.20$ & $4.65 \pm 0.23^{*}$ & $5.01 \pm 0.45$ & $4.99 \pm 0.29$ & $4.89 \pm 0.59$ \\
\hline $\mathrm{Cl}^{-}$ & $\mathrm{mmol} / \mathrm{L}$ & $101.9 \pm 1.1$ & $101.9 \pm 0.8$ & $101.3 \pm 1.1$ & $102.0 \pm 0.9$ & $101.4 \pm 1.3$ \\
\hline
\end{tabular}

Data are expressed as Mean $\pm \mathrm{SD}(\mathrm{n}=10)$. Statistically significant from G1 ( $\left.{ }^{*} p<0.05 ;{ }^{* *} p<0.01\right)$ or G2 $\left({ }^{*} p<0.05 ;{ }^{* \#} p<0.01\right)$. G1: Negative control, G2: Reference control, G3-G5: Test article treatment groups.

ALP, alkaline phosphatase; AST, aspartate aminotransferase; ALT, alanine aminotransferase; CPK: creatinine phosphokinase; TBIL, total bilirubin; GLU, glucose; TCHO, total cholesterol; TG, triglyceride; TP, total protein; ALB, albumin; A/G ratio, albumin/globulin ratio; BUN, blood urea nitrogen; $\mathrm{CRE}$, creatinine; $\mathrm{IP}$, inorganic phosphorus; $\mathrm{Ca}^{2+}$, calcium ion; $\mathrm{Na}^{+}$, sodium ion; $\mathrm{K}^{+}$, potassium ion; $\mathrm{Cl}^{-}$, chloride ion.

0.01). The percentage of LYM, MONO, and EOS of both sexes were significantly decreased $(p<0.05$ or $p<0.01)$, but were considered to changed relative to WBC. Comparison between lenograstim and TS-DP2 groups showed were no statistically significant changes in the main group. Conversely, at a dose of TS-DP2 $1000 \mu \mathrm{g} / \mathrm{kg} / \mathrm{day}$, the WBC and LYM of males were significantly increased $(p<0.05)$ and APTT of females was shorter than that of lenograstim $(p<$ 0.05 ) in the recovery group.
Serum biochemistry test. The statistical significance comparing negative control (G1) in clinical biological chemistry test is shown in Table 5 and 6. ALP in both sexes at all doses was significantly increased $(p<0.01) . \mathrm{Cl}^{-}$and AST (contained females at the dose of $1000 \mu \mathrm{g} / \mathrm{kg} /$ day) of males at all doses was significantly increased $(p<0.05$ or $p<0.01)$. TCHO and $\mathrm{K}^{+}$were significantly decreased $(p<$ 0.05 or $p<0.01$ ) in males, but these values were within the normal reference range provided by Chemon. ALP in both 
Table 6. Serum biochemical findings in 2-week recovery groups with TS-DP2 after 4-week treatment

\begin{tabular}{|c|c|c|c|c|}
\hline \multirow{2}{*}{ Item } & \multirow{2}{*}{ Units } & \multicolumn{3}{|c|}{ Group ( $\mu \mathrm{g} / \mathrm{kg} /$ day) } \\
\hline & & G1 (0) & G2 (1000) & G5 (1000) \\
\hline \multicolumn{5}{|l|}{ Male } \\
\hline AST & $\mathrm{U} / \mathrm{L}$ & $78.1 \pm 9.5$ & $79.5 \pm 14.1$ & $95.3 \pm 12.7^{*}$ \\
\hline ALT & $\mathrm{U} / \mathrm{L}$ & $36.3 \pm 6.8$ & $34.7 \pm 7.2$ & $38.6 \pm 5.4$ \\
\hline ALP & $\mathrm{U} / \mathrm{L}$ & $102.4 \pm 16.4$ & $117.7 \pm 15.7$ & $133.6 \pm 26.5$ \\
\hline CPK & $\mathrm{U} / \mathrm{L}$ & $129.4 \pm 78.1$ & $116.2 \pm 60.0$ & $220.2 \pm 113.2$ \\
\hline TBIL & $\mathrm{mg} / \mathrm{dL}$ & $0.13 \pm 0.00$ & $0.15 \pm 0.01^{* *}$ & $0.15 \pm 0.01^{* * *}$ \\
\hline GLU & $\mathrm{mg} / \mathrm{dL}$ & $111.9 \pm 5.3$ & $98.6 \pm 6.2^{* * *}$ & $98.8 \pm 4.4^{* *}$ \\
\hline TCHO & $\mathrm{mg} / \mathrm{dL}$ & $83.2 \pm 3.6$ & $83.0 \pm 17.8$ & $89.8 \pm 10.3$ \\
\hline TG & $\mathrm{mg} / \mathrm{dL}$ & $38.2 \pm 4.7$ & $38.0 \pm 4.8$ & $36.0 \pm 7.5$ \\
\hline $\mathrm{TP}$ & $\mathrm{g} / \mathrm{dL}$ & $5.59 \pm 0.06$ & $5.62 \pm 0.08$ & $5.55 \pm 0.15$ \\
\hline ALB & $\mathrm{g} / \mathrm{dL}$ & $2.91 \pm 0.04$ & $2.96 \pm 0.07$ & $3.01 \pm 0.07^{*}$ \\
\hline A/Gratio & & $1.08 \pm 0.04$ & $1.12 \pm 0.06$ & $1.19 \pm 0.05^{* *}$ \\
\hline BUN & $\mathrm{mg} / \mathrm{dL}$ & $16.3 \pm 3.3$ & $19.1 \pm 2.7$ & $16.4 \pm 1.3$ \\
\hline CRE & $\mathrm{mg} / \mathrm{dL}$ & $0.45 \pm 0.04$ & $0.45 \pm 0.06$ & $0.45 \pm 0.01$ \\
\hline IP & $\mathrm{mg} / \mathrm{dL}$ & $6.98 \pm 0.39$ & $7.12 \pm 0.39$ & $7.33 \pm 0.33$ \\
\hline $\mathrm{Ca}^{2+}$ & $\mathrm{mg} / \mathrm{dL}$ & $9.60 \pm 0.20$ & $9.46 \pm 0.09$ & $9.49 \pm 0.13$ \\
\hline $\mathrm{Na}^{+}$ & $\mathrm{mmol} / \mathrm{L}$ & $140.8 \pm 1.1$ & $140.7 \pm 0.6$ & $141.3 \pm 1.0$ \\
\hline $\mathrm{K}^{+}$ & $\mathrm{mmol} / \mathrm{L}$ & $4.69 \pm 0.35$ & $4.61 \pm 0.42$ & $4.68 \pm 0.17$ \\
\hline $\mathrm{Cl}^{-}$ & $\mathrm{mmol} / \mathrm{L}$ & $102.6 \pm 1.9$ & $102.8 \pm 1.4$ & $103.0 \pm 1.3$ \\
\hline \multicolumn{5}{|l|}{ Female } \\
\hline AST & $\mathrm{U} / \mathrm{L}$ & $82.4 \pm 10.5$ & $76.9 \pm 4.9$ & $75.2 \pm 5.7$ \\
\hline ALT & $\mathrm{U} / \mathrm{L}$ & $31.0 \pm 2.8$ & $28.6 \pm 1.6$ & $29.4 \pm 3.6$ \\
\hline ALP & $\mathrm{U} / \mathrm{L}$ & $79.5 \pm 15.0$ & $88.5 \pm 17.3$ & $91.0 \pm 24.2$ \\
\hline CPK & $\mathrm{U} / \mathrm{L}$ & $155.8 \pm 85.3$ & $121.4 \pm 42.5$ & $119.6 \pm 56.4$ \\
\hline TBIL & $\mathrm{mg} / \mathrm{dL}$ & $0.15 \pm 0.02$ & $0.17 \pm 0.02$ & $0.17 \pm 0.02$ \\
\hline GLU & $\mathrm{mg} / \mathrm{dL}$ & $107.3 \pm 6.5$ & $104.9 \pm 3.9$ & $102.0 \pm 5.9$ \\
\hline TCHO & $\mathrm{mg} / \mathrm{dL}$ & $88.6 \pm 14.6$ & $73.4 \pm 7.0$ & $71.6 \pm 25.2$ \\
\hline TG & $\mathrm{mg} / \mathrm{dL}$ & $31.8 \pm 4.1$ & $31.0 \pm 5.6$ & $27.8 \pm 3.0$ \\
\hline ТP & $\mathrm{g} / \mathrm{dL}$ & $5.47 \pm 0.19$ & $5.39 \pm 0.10$ & $5.41 \pm 0.16$ \\
\hline ALB & $\mathrm{g} / \mathrm{dL}$ & $3.05 \pm 0.16$ & $3.09 \pm 0.06$ & $3.07 \pm 0.05$ \\
\hline A/Gratio & & $1.26 \pm 0.09$ & $1.34 \pm 0.04$ & $1.32 \pm 0.07$ \\
\hline BUN & $\mathrm{mg} / \mathrm{dL}$ & $19.8 \pm 5.4$ & $18.2 \pm 1.2$ & $19.4 \pm 4.5$ \\
\hline CRE & $\mathrm{mg} / \mathrm{dL}$ & $0.55 \pm 0.06$ & $0.53 \pm 0.02$ & $0.50 \pm 0.04$ \\
\hline IP & $\mathrm{mg} / \mathrm{dL}$ & $6.43 \pm 0.52$ & $6.21 \pm 0.32$ & $6.15 \pm 0.50$ \\
\hline $\mathrm{Ca}^{2+}$ & $\mathrm{mg} / \mathrm{dL}$ & $9.28 \pm 0.15$ & $9.07 \pm 0.21$ & $9.02 \pm 0.12^{*}$ \\
\hline $\mathrm{Na}^{+}$ & $\mathrm{mmol} / \mathrm{L}$ & $139.5 \pm 0.5$ & $140.0 \pm 0.8$ & $139.7 \pm 0.5$ \\
\hline $\mathrm{K}^{+}$ & $\mathrm{mmol} / \mathrm{L}$ & $4.66 \pm 0.23$ & $4.69 \pm 0.28$ & $4.54 \pm 0.26$ \\
\hline $\mathrm{Cl}^{-}$ & $\mathrm{mmol} / \mathrm{L}$ & $102.4 \pm 0.7$ & $105.0 \pm 0.9^{* * *}$ & $105.4 \pm 1.3^{* *}$ \\
\hline
\end{tabular}

Data are expressed as Mean \pm SD $(\mathrm{n}=5)$. Statistically significant from G1 $\left({ }^{*} p<0.05 ;{ }^{* *} p<0.01\right)$ or G2 $\left({ }^{*} p<0.05 ;{ }^{* \#} p<0.01\right)$. G1: Negative control, G2: Reference control, G5: Test article treatment groups.

ALP, alkaline phosphatase; AST, aspartate aminotransferase; ALT, alanine aminotransferase; CPK: creatinine phosphokinase; TBIL, total bilirubin; GLU, glucose; TCHO, total cholesterol; TG, triglyceride; TP, total protein; ALB, albumin; A/G ratio, albumin/globulin ratio; BUN, blood urea nitrogen; $\mathrm{CRE}$, creatinine; $\mathrm{IP}$, inorganic phosphorus; $\mathrm{Ca}^{2+}$, calcium ion; $\mathrm{Na}^{+}$, sodium ion; $\mathrm{K}^{+}$, potassium ion; $\mathrm{Cl}^{-}$, chloride ion.

sexes at the dose of lenograstim $1000 \mathrm{mg} / \mathrm{kg}$ was significantly increased $(p<0.01)$. AST in both sexes, $\mathrm{Na}^{+}$and $\mathrm{Cl}^{-}$ of males, and CRE of females were significantly increased $(p<0.01) . \mathrm{K}^{+}$in both sexes, TP of males, and CPK of females were significantly decreased $(p<0.05$ or $p<0.01)$, but these values were also within the normal reference range provided by Chemon. At a dose of TS-DP2 $1000 \mu \mathrm{g} / \mathrm{kg} / \mathrm{day}$, ALP in males was significantly increased $(p<0.05)$ and CRE in females was significantly decreased $(p<0.01)$ compared to the lenograstim group. In the recovery group, AST, TBIL, $\mathrm{ALB}$, and $\mathrm{A} / \mathrm{G}$ ratio at the dose of $1000 \mu \mathrm{g} / \mathrm{kg} /$ day of males and $\mathrm{Cl}^{-}$of females were significantly increased $(p<0.05$ or $p<0.01)$. GLU of males and $\mathrm{Ca}^{2+}$ of females were significantly decreased $(p<0.05$ or $p<0.01)$. There was no statistical significance between the lenograstim and TS-DP2 groups.

Organ weights. Absolute and relative weights are shown in Table 7, 8, 9, and 10. At all doses, the absolute and 
Table 7. Absolute (g) and relative organ (\%) weights intravenously treated with TS-DP2 for 4 weeks in male rats (main groups)

\begin{tabular}{|c|c|c|c|c|c|}
\hline \multirow{2}{*}{ Organs } & \multicolumn{5}{|c|}{ Group $(\mu \mathrm{g} / \mathrm{kg} /$ day) } \\
\hline & G1 (0) & G2 (1000) & G3 (250) & G4 (500) & G5 (1000) \\
\hline Body weights & $301.69 \pm 24.86$ & $271.86 \pm 28.88^{*}$ & $284.81 \pm 9.63$ & $283.09 \pm 13.77$ & $274.96 \pm 24.22$ \\
\hline Adrenal gland-left & $0.0249 \pm 0.0044$ & $0.0243 \pm 0.0023$ & $0.0232 \pm 0.0034$ & $0.0227 \pm 0.0025$ & $0.0218 \pm 0.0017^{\#}$ \\
\hline$\%$ to body weight & $0.0082 \pm 0.0011$ & $0.0089 \pm 0.0006$ & $0.0081 \pm 0.0011$ & $0.0080 \pm 0.0008$ & $0.0079 \pm 0.0006^{\# \#}$ \\
\hline Adrenal gland-right & $0.0252 \pm 0.0034$ & $0.0231 \pm 0.0014$ & $0.0221 \pm 0.0028$ & $0.0230 \pm 0.0029$ & $0.0220 \pm 0.0024$ \\
\hline$\%$ to body weight & $0.0084 \pm 0.0009$ & $0.0086 \pm 0.0008$ & $0.0078 \pm 0.0009$ & $0.0081 \pm 0.0009$ & $0.0081 \pm 0.0012$ \\
\hline Pituitary gland & $0.0098 \pm 0.0009$ & $0.0090 \pm 0.0012$ & $0.0096 \pm 0.0009$ & $0.0095 \pm 0.0008$ & $0.0090 \pm 0.0008$ \\
\hline$\%$ to body weight & $0.0032 \pm 0.0003$ & $0.0033 \pm 0.0004$ & $0.0034 \pm 0.0003$ & $0.0033 \pm 0.0003$ & $0.0033 \pm 0.0002$ \\
\hline Thymus & $0.5490 \pm 0.1005$ & $0.4173 \pm 0.0616^{* *}$ & $0.4409 \pm 0.0852^{*}$ & $0.4657 \pm 0.0726^{*}$ & $0.4345 \pm 0.0926^{*}$ \\
\hline$\%$ to body weight & $0.1815 \pm 0.0276$ & $0.1532 \pm 0.0123^{*}$ & $0.1547 \pm 0.0287$ & $0.1643 \pm 0.0229$ & $0.1576 \pm 0.0284$ \\
\hline Prostate gland & $0.3957 \pm 0.0872$ & $0.3594 \pm 0.0972$ & $0.3578 \pm 0.0534$ & $0.3725 \pm 0.0791$ & $0.3988 \pm 0.1033$ \\
\hline$\%$ to body weight & $0.1316 \pm 0.0302$ & $0.1312 \pm 0.0286$ & $0.1260 \pm 0.0203$ & $0.1319 \pm 0.0289$ & $0.1444 \pm 0.0323$ \\
\hline Testis-left & $1.8273 \pm 0.2179$ & $1.8570 \pm 0.1246$ & $1.8433 \pm 0.1700$ & $1.8443 \pm 0.1014$ & $1.7569 \pm 0.1236$ \\
\hline$\%$ to body weight & $0.6073 \pm 0.0698$ & $0.6881 \pm 0.0646^{*}$ & $0.6466 \pm 0.0476$ & $0.6524 \pm 0.0400$ & $0.6413 \pm 0.0465$ \\
\hline Testis-right & $1.7898 \pm 0.4889$ & $1.8266 \pm 0.1167$ & $1.8630 \pm 0.1681$ & $1.8183 \pm 0.1324$ & $1.7920 \pm 0.0865$ \\
\hline$\%$ to body weight & $0.5892 \pm 0.1501$ & $0.6782 \pm 0.0763$ & $0.6536 \pm 0.0477$ & $0.6431 \pm 0.0489$ & $0.6551 \pm 0.0495$ \\
\hline Epididymis-left & $0.4581 \pm 0.0489$ & $0.4658 \pm 0.0270$ & $0.4563 \pm 0.0331$ & $0.4758 \pm 0.0345$ & $0.4481 \pm 0.0312$ \\
\hline$\%$ to body weight & $0.1525 \pm 0.0183$ & $0.1726 \pm 0.0149^{*}$ & $0.1601 \pm 0.0085$ & $0.1681 \pm 0.0098$ & $0.1636 \pm 0.0125$ \\
\hline Epididymis-right & $0.4684 \pm 0.0576$ & $0.4675 \pm 0.0303$ & $0.4638 \pm 0.0261$ & $0.4777 \pm 0.0396$ & $0.4526 \pm 0.0350$ \\
\hline$\%$ to body weight & $0.1555 \pm 0.0186$ & $0.1733 \pm 0.0168^{*}$ & $0.1628 \pm 0.0063$ & $0.1691 \pm 0.0158$ & $0.1653 \pm 0.0151$ \\
\hline Spleen & $0.7549 \pm 0.0823$ & $1.9607 \pm 0.3182^{* *}$ & $1.1833 \pm 0.1065^{* *}$ & $1.5831 \pm 0.2794^{* *}$ & $1.8640 \pm 0.3009^{* *}$ \\
\hline$\%$ to body weight & $0.2508 \pm 0.0266$ & $0.7219 \pm 0.0918^{* *}$ & $0.4152 \pm 0.0308^{* * *}$ & $0.5573 \pm 0.0787^{* *}$ & $0.6780 \pm 0.0874^{* * *}$ \\
\hline Kidney-left & $1.0822 \pm 0.0863$ & $0.9402 \pm 0.1002^{* *}$ & $0.9592 \pm 0.0705^{* *}$ & $0.9560 \pm 0.0639^{* * *}$ & $0.9432 \pm 0.0800^{* * *}$ \\
\hline$\%$ to body weight & $0.3591 \pm 0.0165$ & $0.3462 \pm 0.0202$ & $0.3366 \pm 0.0188^{*}$ & $0.3379 \pm 0.0199^{*}$ & $0.3434 \pm 0.0149$ \\
\hline Kidney-right & $1.1027 \pm 0.0832$ & $0.9428 \pm 0.0932^{* *}$ & $0.9889 \pm 0.0490^{* *}$ & $0.9914 \pm 0.0577^{* * *}$ & $0.9796 \pm 0.0755^{* *}$ \\
\hline$\%$ to body weight & $0.3662 \pm 0.0210$ & $0.3481 \pm 0.0303$ & $0.3473 \pm 0.0156$ & $0.3505 \pm 0.0180$ & $0.3569 \pm 0.0164$ \\
\hline Heart & $1.1057 \pm 0.1298$ & $1.0172 \pm 0.1198$ & $1.0228 \pm 0.0707$ & $1.0254 \pm 0.1221$ & $0.9755 \pm 0.0610^{*}$ \\
\hline$\%$ to body weight & $0.3662 \pm 0.0268$ & $0.3742 \pm 0.0205$ & $0.3591 \pm 0.0218$ & $0.3615 \pm 0.0290$ & $0.3558 \pm 0.0174^{\#}$ \\
\hline Lung & $1.3675 \pm 0.1158$ & $1.4967 \pm 0.1422^{*}$ & $1.3920 \pm 0.0858$ & $1.4084 \pm 0.0967$ & $1.4962 \pm 0.1322$ \\
\hline$\%$ to body weight & $0.4543 \pm 0.0339$ & $0.5531 \pm 0.0489^{* * *}$ & $0.4886 \pm 0.0218^{*}$ & $0.4975 \pm 0.0244^{*}$ & $0.5464 \pm 0.0515^{* *}$ \\
\hline Brain & $1.8619 \pm 0.1295$ & $1.7813 \pm 0.0489$ & $1.8156 \pm 0.0894$ & $1.7544 \pm 0.0503$ & $1.7903 \pm 0.1038$ \\
\hline$\%$ to body weight & $0.6185 \pm 0.0344$ & $0.6616 \pm 0.0677$ & $0.6377 \pm 0.0274$ & $0.6209 \pm 0.0332$ & $0.6542 \pm 0.0492$ \\
\hline Liver & $8.6418 \pm 0.8740$ & $7.7821 \pm 1.1204$ & $7.8926 \pm 0.6309$ & $7.9749 \pm 0.7722$ & $7.7915 \pm 0.8519$ \\
\hline$\%$ to body weight & $2.8628 \pm 0.1222$ & $2.8538 \pm 0.1476$ & $2.7696 \pm 0.1760$ & $2.8134 \pm 0.1623$ & $2.8320 \pm 0.1510$ \\
\hline
\end{tabular}

The body weights were measured right before necropsy after overnight fasting. Data are expressed as Mean \pm SD $(n=10)$. Statistically significant from G1 $\left({ }^{*} p<0.05 ;{ }^{* *} p<0.01\right)$ or G2 $\left({ }^{*} p<0.05 ;{ }^{\# \#} p<0.01\right)$.

G1: Negative control, G2: Reference control, G3-G5: Test article treatment groups.

Table 8. Absolute (g) and relative organ (\%) weights intravenously treated with TS-DP2 for 4 weeks in female rats (main groups)

\begin{tabular}{llcccc}
\hline \hline \multirow{2}{*}{ Organs } & \multicolumn{5}{c}{ Group $(\mu \mathrm{g} / \mathrm{kg} /$ day $)$} \\
\cline { 2 - 6 } & $\mathrm{G} 1(0)$ & $\mathrm{G} 2(1000)$ & $\mathrm{G} 3(250)$ & $\mathrm{G} 4(500)$ & $\mathrm{G})(1000)$ \\
\hline Body weights & $190.42 \pm 8.20$ & $193.64 \pm 10.43$ & $192.10 \pm 11.30$ & $190.66 \pm 6.22$ & $190.53 \pm 17.23$ \\
Ovary-left & $0.0393 \pm 0.0077$ & $0.0407 \pm 0.0082$ & $0.0393 \pm 0.0066$ & $0.0393 \pm 0.0050$ & $0.0387 \pm 0.0060$ \\
$\quad \%$ to body weight & $0.0206 \pm 0.0036$ & $0.0211 \pm 0.0048$ & $0.0205 \pm 0.0035$ & $0.0206 \pm 0.0026$ & $0.0204 \pm 0.0035$ \\
Ovary-right & $0.0395 \pm 0.0071$ & $0.0417 \pm 0.0084$ & $0.0358 \pm 0.0080$ & $0.0375 \pm 0.0068$ & $0.0381 \pm 0.0089$ \\
$\quad \%$ to body weight & $0.0207 \pm 0.0031$ & $0.0216 \pm 0.0045$ & $0.0186 \pm 0.0038$ & $0.0196 \pm 0.0034$ & $0.0200 \pm 0.0041$ \\
Adrenal gland-left & $0.0278 \pm 0.0027$ & $0.0296 \pm 0.0040$ & $0.0293 \pm 0.0043$ & $0.0283 \pm 0.0039$ & $0.0295 \pm 0.0028$ \\
$\quad \%$ to body weight & $0.0146 \pm 0.0013$ & $0.0153 \pm 0.0022$ & $0.0152 \pm 0.0018$ & $0.0148 \pm 0.0019$ & $0.0156 \pm 0.0019$ \\
Adrenal gland-right & $0.0274 \pm 0.0020$ & $0.0280 \pm 0.0041$ & $0.0288 \pm 0.0033$ & $0.0286 \pm 0.0034$ & $0.0279 \pm 0.0019$ \\
$\quad \%$ to body weight & $0.0144 \pm 0.0008$ & $0.0145 \pm 0.0021$ & $0.0150 \pm 0.0014$ & $0.0150 \pm 0.0015$ & $0.0147 \pm 0.0012$ \\
Pituitary gland & $0.0111 \pm 0.0014$ & $0.0112 \pm 0.0012$ & $0.0110 \pm 0.0013$ & $0.0104 \pm 0.0012$ & $0.0106 \pm 0.0013$ \\
$\quad \%$ to body weight & $0.0058 \pm 0.0009$ & $0.0058 \pm 0.0006$ & $0.0057 \pm 0.0007$ & $0.0055 \pm 0.0007$ & $0.0056 \pm 0.0005$ \\
Thymus & $0.3723 \pm 0.0560$ & $0.3371 \pm 0.0687$ & $0.3172 \pm 0.0615$ & $0.3589 \pm 0.0533$ & $0.3489 \pm 0.0516$ \\
$\quad \%$ to body weight & $0.1953 \pm 0.0272$ & $0.1750 \pm 0.0390$ & $0.1648 \pm 0.0294$ & $0.1880 \pm 0.0251$ & $0.1826 \pm 0.0161$ \\
Uterus & $0.5515 \pm 0.2805$ & $0.5473 \pm 0.1836$ & $0.3970 \pm 0.1240$ & $0.6834 \pm 0.3254$ & $0.4347 \pm 0.1139$ \\
$\quad \%$ to body weight & $0.2893 \pm 0.1457$ & $0.2821 \pm 0.0907$ & $0.2071 \pm 0.0639$ & $0.3571 \pm 0.1673$ & $0.2308 \pm 0.0684$ \\
\hline
\end{tabular}


Table 8. Continued

\begin{tabular}{cccccc}
\hline \hline \multirow{2}{*}{ Organs } & \multicolumn{5}{c}{ Group $(\mu \mathrm{g} / \mathrm{kg} / \mathrm{day})$} \\
\cline { 2 - 6 } & $\mathrm{G} 1(0)$ & $\mathrm{G} 2(1000)$ & $\mathrm{G} 3(250)$ & $\mathrm{G} 4(500)$ & $\mathrm{G})(1000)$ \\
\hline Spleen & $0.5431 \pm 0.0508$ & $1.2210 \pm 0.1438^{* * *}$ & $0.8342 \pm 0.1093^{* * *}$ & $0.9850 \pm 0.0920^{* *}$ & $1.2014 \pm 0.2492^{* * *}$ \\
\% to body weight & $0.2850 \pm 0.0201$ & $0.6296 \pm 0.0572^{* *}$ & $0.4346 \pm 0.0547^{* *}$ & $0.5176 \pm 0.0568^{* *}$ & $0.6268 \pm 0.0879^{* *}$ \\
Kidney-left & $0.6397 \pm 0.0385$ & $0.6410 \pm 0.0476$ & $0.6385 \pm 0.0347$ & $0.6412 \pm 0.0346$ & $0.6558 \pm 0.0481$ \\
\% to body weight & $0.3359 \pm 0.0146$ & $0.3311 \pm 0.0179$ & $0.3325 \pm 0.0084$ & $0.3365 \pm 0.0182$ & $0.3454 \pm 0.0235$ \\
Kidney-right & $0.6719 \pm 0.0402$ & $0.6629 \pm 0.0372$ & $0.6521 \pm 0.0432$ & $0.6566 \pm 0.0407$ & $0.6605 \pm 0.0554$ \\
\% to body weight & $0.3530 \pm 0.0176$ & $0.3429 \pm 0.0214$ & $0.3395 \pm 0.0135$ & $0.3446 \pm 0.0212$ & $0.3477 \pm 0.0269$ \\
Heart & $0.7258 \pm 0.0449$ & $0.7323 \pm 0.0569$ & $0.7569 \pm 0.0502$ & $0.7479 \pm 0.0468$ & $0.7476 \pm 0.1086$ \\
\% to body weight & $0.3816 \pm 0.0265$ & $0.3782 \pm 0.0211$ & $0.3945 \pm 0.0244$ & $0.3926 \pm 0.0270$ & $0.3919 \pm 0.0361$ \\
Lung & $1.0922 \pm 0.0793$ & $1.1791 \pm 0.0630^{*}$ & $1.1067 \pm 0.0595$ & $1.1049 \pm 0.0432$ & $1.2008 \pm 0.0724^{* *}$ \\
\% to body weight & $0.5737 \pm 0.0342$ & $0.6092 \pm 0.0185^{*}$ & $0.5765 \pm 0.0191$ & $0.5798 \pm 0.0226$ & $0.6335 \pm 0.0535$ \\
Brain & $1.7405 \pm 0.0650$ & $1.6890 \pm 0.0867$ & $1.6656 \pm 0.0693$ & $1.6892 \pm 0.0695$ & $1.6675 \pm 0.0650$ \\
\% to body weight & $0.9151 \pm 0.0421$ & $0.8743 \pm 0.0605$ & $0.8703 \pm 0.0718$ & $0.8861 \pm 0.0272$ & $0.8813 \pm 0.0823$ \\
Liver & $5.1819 \pm 0.3296$ & $5.5505 \pm 0.2384^{*}$ & $5.3834 \pm 0.4429$ & $5.3964 \pm 0.2108$ & $5.5037 \pm 0.6615$ \\
\% to body weight & $2.7227 \pm 0.1609$ & $2.8701 \pm 0.1173^{*}$ & $2.8001 \pm 0.1150$ & $2.8315 \pm 0.1019$ & $2.8852 \pm 0.1578$ \\
\hline
\end{tabular}

The body weights were measured right before necropsy after overnight fasting. Data are expressed as Mean \pm SD $(n=10)$. Statistically significant from G1 ( $\left.{ }^{*} p<0.05 ;{ }^{* *} p<0.01\right)$ or G2 $\left({ }^{*} p<0.05 ;{ }^{\# \#} p<0.01\right)$.

G1: Negative control, G2: Reference control, G3-G5: Test article treatment groups.

Table 9. Absolute (g) and relative organ (\%) weights intravenously treated with TS-DP2 for 4 weeks in male rats (recovery groups)

\begin{tabular}{|c|c|c|c|}
\hline \multirow{2}{*}{ Organs } & \multicolumn{3}{|c|}{ Group ( $\mu \mathrm{g} / \mathrm{kg} /$ day) } \\
\hline & G1 (0) & G2 (1000) & G5 (1000) \\
\hline Body weights & $337.73 \pm 21.45$ & $299.88 \pm 25.27^{*}$ & $317.96 \pm 22.79$ \\
\hline Adrenal gland-left & $0.0253 \pm 0.0024$ & $0.0261 \pm 0.0029$ & $0.0252 \pm 0.0027$ \\
\hline$\%$ to body weight & $0.0075 \pm 0.0010$ & $0.0087 \pm 0.0012$ & $0.0080 \pm 0.0011$ \\
\hline Adrenal gland-right & $0.0232 \pm 0.0038$ & $0.0239 \pm 0.0012$ & $0.0243 \pm 0.0032$ \\
\hline$\%$ to body weight & $0.0069 \pm 0.0012$ & $0.0080 \pm 0.0007$ & $0.0077 \pm 0.0012$ \\
\hline Pituitary gland & $0.0109 \pm 0.0008$ & $0.0105 \pm 0.0008$ & $0.0111 \pm 0.0013$ \\
\hline$\%$ to body weight & $0.0032 \pm 0.0002$ & $0.0035 \pm 0.0004$ & $0.0035 \pm 0.0002$ \\
\hline Thymus & $0.4752 \pm 0.0623$ & $0.3509 \pm 0.1011^{*}$ & $0.3963 \pm 0.0687$ \\
\hline$\%$ to body weight & $0.1405 \pm 0.0143$ & $0.1162 \pm 0.0277$ & $0.1247 \pm 0.0208$ \\
\hline Prostate gland & $0.4786 \pm 0.0956$ & $0.3963 \pm 0.1029$ & $0.4228 \pm 0.0511$ \\
\hline$\%$ to body weight & $0.1418 \pm 0.0269$ & $0.1337 \pm 0.0421$ & $0.1342 \pm 0.0228$ \\
\hline Testis-left & $1.8603 \pm 0.0980$ & $1.8281 \pm 0.2295$ & $1.8254 \pm 0.1419$ \\
\hline$\%$ to body weight & $0.5522 \pm 0.0383$ & $0.6092 \pm 0.0543$ & $0.5757 \pm 0.0531$ \\
\hline Testis-right & $1.8606 \pm 0.1165$ & $1.7769 \pm 0.2417$ & $1.7787 \pm 0.1462$ \\
\hline$\%$ to body weight & $0.5520 \pm 0.0387$ & $0.5918 \pm 0.0611$ & $0.5603 \pm 0.0442$ \\
\hline Epididymis-left & $0.5731 \pm 0.0434$ & $0.5351 \pm 0.0580$ & $0.5433 \pm 0.0359$ \\
\hline$\%$ to body weight & $0.1702 \pm 0.0168$ & $0.1784 \pm 0.0128$ & $0.1713 \pm 0.0134$ \\
\hline Epididymis-right & $0.5540 \pm 0.0297$ & $0.5186 \pm 0.0658$ & $0.5364 \pm 0.0450$ \\
\hline$\%$ to body weight & $0.1645 \pm 0.0128$ & $0.1726 \pm 0.0123$ & $0.1689 \pm 0.0124$ \\
\hline Spleen & $0.8227 \pm 0.2058$ & $0.8263 \pm 0.1734$ & $0.9894 \pm 0.1334$ \\
\hline$\%$ to body weight & $0.2421 \pm 0.0497$ & $0.2741 \pm 0.0420$ & $0.3115 \pm 0.0401^{*}$ \\
\hline Kidney-left & $1.1416 \pm 0.0821$ & $1.0138 \pm 0.1385$ & $1.0390 \pm 0.1108$ \\
\hline$\%$ to body weight & $0.3382 \pm 0.0151$ & $0.3371 \pm 0.0267$ & $0.3262 \pm 0.0125$ \\
\hline Kidney-right & $1.0596 \pm 0.1815$ & $1.0551 \pm 0.1490$ & $1.0548 \pm 0.0641$ \\
\hline$\%$ to body weight & $0.3154 \pm 0.0591$ & $0.3505 \pm 0.0242$ & $0.3323 \pm 0.0180$ \\
\hline Heart & $1.1478 \pm 0.0381$ & $1.0513 \pm 0.1186$ & $1.0665 \pm 0.0824$ \\
\hline$\%$ to body weight & $0.3411 \pm 0.0260$ & $0.3500 \pm 0.0140$ & $0.3354 \pm 0.0112$ \\
\hline Lung & $1.4830 \pm 0.1304$ & $1.2773 \pm 0.1494^{*}$ & $1.4490 \pm 0.1233$ \\
\hline$\%$ to body weight & $0.4395 \pm 0.0333$ & $0.4252 \pm 0.0241$ & $0.4562 \pm 0.0326$ \\
\hline Brain & $1.8181 \pm 0.0748$ & $1.7656 \pm 0.1512$ & $1.7807 \pm 0.0735$ \\
\hline$\%$ to body weight & $0.5404 \pm 0.0473$ & $0.5893 \pm 0.0304$ & $0.5616 \pm 0.0339$ \\
\hline Liver & $9.0080 \pm 0.8222$ & $7.9560 \pm 0.8633$ & $8.3609 \pm 0.9597$ \\
\hline$\%$ to body weight & $2.6654 \pm 0.1462$ & $2.6501 \pm 0.1239$ & $2.6248 \pm 0.1373$ \\
\hline
\end{tabular}

The body weights were measured right before necropsy after overnight fasting. Data are expressed as Mean \pm SD $(n=5)$. Statistically significant from G2 ( $\left.{ }^{\#} p<0.05 ;{ }^{\# \#} p<0.01\right)$. G1: Negative control, G2: Reference control, G5: Test article treatment groups. 
Table 10. Absolute (g) and relative organ (\%) weights intravenously treated with TS-DP2 for 4 weeks in female rats (recovery groups)

\begin{tabular}{|c|c|c|c|}
\hline \multirow{2}{*}{ Organs } & \multicolumn{3}{|c|}{ Group $(\mu \mathrm{g} / \mathrm{kg} /$ day) } \\
\hline & G1 (0) & G2 (1000) & G5 (1000) \\
\hline Body weights & $209.45 \pm 8.71$ & $208.84 \pm 5.65$ & $208.75 \pm 13.50$ \\
\hline Ovary-left & $0.0371 \pm 0.0042$ & $0.0362 \pm 0.0056$ & $0.0377 \pm 0.0073$ \\
\hline$\%$ to body weight & $0.0177 \pm 0.0013$ & $0.0174 \pm 0.0028$ & $0.0180 \pm 0.0030$ \\
\hline Ovary-right & $0.0360 \pm 0.0083$ & $0.0400 \pm 0.0067$ & $0.0425 \pm 0.0084$ \\
\hline$\%$ to body weight & $0.0171 \pm 0.0036$ & $0.0192 \pm 0.0034$ & $0.0203 \pm 0.0032$ \\
\hline Adrenal gland-left & $0.0303 \pm 0.0036$ & $0.0328 \pm 0.0036$ & $0.0284 \pm 0.0046$ \\
\hline$\%$ to body weight & $0.0144 \pm 0.0013$ & $0.0157 \pm 0.0016$ & $0.0136 \pm 0.0018$ \\
\hline Adrenal gland-right & $0.0297 \pm 0.0017$ & $0.0318 \pm 0.0021$ & $0.0284 \pm 0.0048$ \\
\hline$\%$ to body weight & $0.0142 \pm 0.0004$ & $0.0152 \pm 0.0010$ & $0.0137 \pm 0.0026$ \\
\hline Pituitary gland & $0.0120 \pm 0.0014$ & $0.0114 \pm 0.0009$ & $0.0116 \pm 0.0015$ \\
\hline$\%$ to body weight & $0.0058 \pm 0.0007$ & $0.0055 \pm 0.0003$ & $0.0056 \pm 0.0006$ \\
\hline Thymus & $0.2964 \pm 0.0713$ & $0.2990 \pm 0.0258$ & $0.3133 \pm 0.0558$ \\
\hline$\%$ to body weight & $0.1410 \pm 0.0300$ & $0.1431 \pm 0.0104$ & $0.1493 \pm 0.0197$ \\
\hline Uterus & $0.5213 \pm 0.2714$ & $0.6488 \pm 0.1597$ & $0.6882 \pm 0.2862$ \\
\hline$\%$ to body weight & $0.2464 \pm 0.1192$ & $0.3095 \pm 0.0687$ & $0.3324 \pm 0.1400$ \\
\hline Spleen & $0.5499 \pm 0.0381$ & $0.6628 \pm 0.0576^{* *}$ & $0.6445 \pm 0.0643^{*}$ \\
\hline$\%$ to body weight & $0.2626 \pm 0.0143$ & $0.3178 \pm 0.0321^{*}$ & $0.3093 \pm 0.0317^{*}$ \\
\hline Kidney-left & $0.6724 \pm 0.0575$ & $0.6576 \pm 0.0432$ & $0.6883 \pm 0.0325$ \\
\hline$\%$ to body weight & $0.3207 \pm 0.0167$ & $0.3149 \pm 0.0198$ & $0.3303 \pm 0.0165$ \\
\hline Kidney-right & $0.6896 \pm 0.0558$ & $0.6846 \pm 0.0372$ & $0.6890 \pm 0.0270$ \\
\hline$\%$ to body weight & $0.3290 \pm 0.0166$ & $0.3279 \pm 0.0178$ & $0.3310 \pm 0.0222$ \\
\hline Heart & $0.7505 \pm 0.0699$ & $0.7788 \pm 0.0745$ & $0.7557 \pm 0.0750$ \\
\hline$\%$ to body weight & $0.3583 \pm 0.0288$ & $0.3734 \pm 0.0406$ & $0.3615 \pm 0.0157$ \\
\hline Lung & $1.1136 \pm 0.0749$ & $1.1376 \pm 0.0471$ & $1.1128 \pm 0.0488$ \\
\hline$\%$ to body weight & $0.5317 \pm 0.0282$ & $0.5453 \pm 0.0324$ & $0.5344 \pm 0.0330$ \\
\hline Brain & $1.7107 \pm 0.0380$ & $1.7451 \pm 0.1381$ & $1.7477 \pm 0.0480$ \\
\hline$\%$ to body weight & $0.8177 \pm 0.0338$ & $0.8367 \pm 0.0775$ & $0.8398 \pm 0.0535$ \\
\hline Liver & $5.4755 \pm 0.4339$ & $5.4061 \pm 0.2026$ & $5.2574 \pm 0.4371$ \\
\hline$\%$ to body weight & $2.6131 \pm 0.1556$ & $2.5891 \pm 0.0831$ & $2.5181 \pm 0.1168$ \\
\hline
\end{tabular}

The body weights were measured right before necropsy after overnight fasting. Data are expressed as Mean \pm SD $(n=5)$. Statistically significant from G2 ( $\left.{ }^{\sharp} p<0.05 ;{ }^{\# \#} p<0.01\right)$. G1: Negative control, G2: Reference control, G5: Test article treatment groups.

relative weights of the spleen in both sexes were significantly higher than negative control (G1) $(p<0.01)$. At the dose of $1000 \mu \mathrm{g} / \mathrm{kg} /$ day, the absolute weight of heart of males was also significantly lower $(p<0.05)$ and lung of females was significantly higher $(p<0.01)$. In males, the absolute weight of both kidneys at all doses and the relative weight of left kidney at doses of 250 and $500 \mu \mathrm{g} / \mathrm{kg} /$ day were significantly decreased $(p<0.05$ or $p<0.01)$. In addition, at all doses, the relative weight of lung was significantly higher in males $(p<0.05$ or $p<0.01)$ and the absolute weight of thymus was significantly decreased $(p<0.05)$. In the lenograstim group, lower fasting body weight was considered to be a source of the observed changes. Fasting body weight of males was significantly lower $(p<0.05)$ and the absolute and relative weights of liver of females were significantly increased $(p<0.05)$. The absolute and relative weights of spleen and lung of both sexes were significantly increased $(p<0.05$ or $p<0.01)$.

In addition, the relative weight of the left testis and both epididymidis was significantly increased $(p<0.05)$, and the relative weight of both kidneys and the absolute and rela- tive weights of thymus of males were significantly decreased ( $p<0.05$ or $p<0.01)$. Decreased fasting body weight was again considered to be a source of the observed changes. At the dose of $1000 \mu \mathrm{g} / \mathrm{kg} /$ day of TS-DP2, the absolute and relative weights of the left adrenal gland and the relative weight of hearts in males were significantly decreased compared to the lenograstim group ( $p<0.05$ or $p<0.01)$. There was no statistically significant change in the recovery group.

Necropsy findings. Main necropsy findings are shown in Table 11. The increased size of the spleen was observed at all doses in males and in 3, 3, and 9 cases in females at doses of 250,500 , and $1000 \mu \mathrm{g} / \mathrm{kg} / \mathrm{day}$, respectively. Hind limb edema was observed in 2 cases in males and 1 case in females at the dose of TS-DP2 $1000 \mu \mathrm{g} / \mathrm{kg} / \mathrm{day}$. In the lenograstim group, the increased size of spleen was observed in all cases in males and in 8 cases in females, and hind limb edema was observed in 3 cases in males. In the recovery groups, the increased size of spleen was seen in 2 cases and the hind limb edema in 1 case of males at the dose of $1000 \mu \mathrm{g} / \mathrm{kg} /$ day of TS-DP2. There were no changes 
Table 11. Necropsy findings intravenously treated with TS-DP2 for 4 weeks in rats

\begin{tabular}{|c|c|c|c|c|c|c|c|c|c|}
\hline \multirow[b]{2}{*}{ Organs } & \multirow[b]{2}{*}{ Findings } & \multicolumn{5}{|c|}{ Main group $(\mu \mathrm{g} / \mathrm{kg} /$ day $)$} & \multicolumn{3}{|c|}{ Recovery group ( $\mu \mathrm{g} / \mathrm{kg} /$ day) } \\
\hline & & $\begin{array}{l}\mathrm{G} 1 \\
(0)\end{array}$ & $\begin{array}{c}\mathrm{G} 2 \\
(1000)\end{array}$ & $\begin{array}{c}\mathrm{G} 3 \\
(250)\end{array}$ & $\begin{array}{c}\mathrm{G} 4 \\
(500)\end{array}$ & $\begin{array}{c}\mathrm{G} 5 \\
(1000)\end{array}$ & $\begin{array}{l}\text { G1 } \\
(0)\end{array}$ & $\begin{array}{c}\mathrm{G} 2 \\
(1000)\end{array}$ & $\begin{array}{c}\text { G5 } \\
(1000)\end{array}$ \\
\hline \multicolumn{10}{|l|}{ Male } \\
\hline \multicolumn{2}{|c|}{ No gross findings } & $9 / 10$ & $0 / 10$ & $0 / 10$ & $0 / 10$ & $0 / 10$ & $5 / 5$ & $5 / 5$ & $3 / 5$ \\
\hline Appearance & Hindlimb edema & $0 / 10$ & $3 / 10$ & $0 / 10$ & $0 / 10$ & $2 / 10$ & $0 / 5$ & $0 / 5$ & $1 / 5$ \\
\hline Testis & Decreased size of right part & $1 / 10$ & $0 / 10$ & $0 / 10$ & $0 / 10$ & $0 / 10$ & $0 / 5$ & $0 / 5$ & $0 / 5$ \\
\hline Epididymis & Decreased size of right part & $1 / 10$ & $0 / 10$ & $0 / 10$ & $0 / 10$ & $0 / 10$ & $0 / 5$ & $0 / 5$ & $0 / 5$ \\
\hline Spleen & Increased size & $0 / 10$ & $10 / 10$ & $10 / 10$ & $10 / 10$ & $10 / 10$ & $0 / 5$ & $0 / 5$ & $2 / 5$ \\
\hline \multirow[t]{2}{*}{ Liver } & $\begin{array}{l}\text { Partially dark red discoloration } \\
\text { of left lobe }\end{array}$ & $0 / 10$ & $1 / 10$ & $0 / 10$ & $0 / 10$ & $0 / 10$ & $0 / 5$ & $0 / 5$ & $0 / 5$ \\
\hline & $\begin{array}{l}\text { Partially white discoloration } \\
\text { of caudate lobe }\end{array}$ & $0 / 10$ & $0 / 10$ & $0 / 10$ & $1 / 10$ & $0 / 10$ & $0 / 5$ & $0 / 5$ & $0 / 5$ \\
\hline \multicolumn{10}{|l|}{ Female } \\
\hline \multicolumn{2}{|c|}{ No gross findings } & $8 / 10$ & $1 / 10$ & $7 / 10$ & $5 / 10$ & $1 / 10$ & $4 / 5$ & $3 / 5$ & $3 / 5$ \\
\hline Appearance & Hindlimb edema & $0 / 10$ & $0 / 10$ & $0 / 10$ & $0 / 10$ & $1 / 10$ & $0 / 5$ & $0 / 5$ & $0 / 5$ \\
\hline \multirow[t]{2}{*}{ Uterus } & Retention of clear fluid & $2 / 10$ & $4 / 10$ & $0 / 10$ & $3 / 10$ & $1 / 10$ & $1 / 5$ & $2 / 5$ & $2 / 5$ \\
\hline & $\begin{array}{l}\text { Retention of clear fluid and cyst } \\
\text { formation included retention } \\
\text { of clear fluid on uterine horn }(0.1 \mathrm{~cm})\end{array}$ & $0 / 10$ & $0 / 10$ & $0 / 10$ & $1 / 10$ & $0 / 10$ & $0 / 5$ & $0 / 5$ & $0 / 5$ \\
\hline Spleen & Increased size & $0 / 10$ & $8 / 10$ & $3 / 10$ & $3 / 10$ & $9 / 10$ & $0 / 5$ & $0 / 5$ & $0 / 5$ \\
\hline Liver & $\begin{array}{l}\text { Light yellow discoloration } \\
\text { on the central of left lobe }(0.3 \mathrm{~cm})\end{array}$ & $0 / 10$ & $1 / 10$ & $0 / 10$ & $0 / 10$ & $0 / 10$ & $0 / 5$ & $0 / 5$ & $0 / 5$ \\
\hline Adrenal gland & $\begin{array}{l}\text { Increased size }\left(0.5^{*} 0.3 \mathrm{~cm}\right) \\
\text { and dark red discoloration }\end{array}$ & $0 / 10$ & $0 / 10$ & $1 / 10$ & $0 / 10$ & $0 / 10$ & $0 / 5$ & $0 / 5$ & $0 / 5$ \\
\hline
\end{tabular}

Data represented number of animal which gross finding was observed/total animal number in the group.

in recovery group of TS-DP2. There were no any other toxicologically meaningful changes in either group in the necropsy findings.

Histopathological examinations. The important lesions were the signs observed on liver, spleen, femur, sternum, hind limb, and tartus (Table 12 and 13) (Fig. 4, 5 and 6). In the main groups, the important lesion observed on the liver was infiltration of mononuclear cell and the increase of erythroid and myeloid extramedullary hematopoiesis, occurring in a dose-dependent manner. The erythroid and myeloid extramedullary hematopoiesis were significantly higher at all TS-DP2 doses and lenograstim for both sexes compared to negative control ( $p<0.05$ or $p<0.01$ or $p<0.001)$. The main lesion observed on spleen was due to the increase of erythroid and myeloid extramedullary hematopoiesis. These lesions were significantly higher at all TS-DP2 doses and the lenograstim groups in both sexes $(p<0.05$ or $p<0.01$

Table 12. Histopathologic findings in the bone marrow and femorotibial joint

\begin{tabular}{|c|c|c|c|c|c|c|c|c|c|}
\hline \multirow{3}{*}{ Organs } & \multirow{2}{*}{$\begin{array}{c}\text { Groups } \\
\text { Findings }\end{array}$} & \multicolumn{5}{|c|}{ Main } & \multicolumn{3}{|c|}{ Recovery } \\
\hline & & G1 & $\mathrm{G} 2$ & $\mathrm{G} 3$ & G4 & G5 & G1 & $\mathrm{G} 2$ & G5 \\
\hline & Number examined & 10 & 10 & 10 & 10 & 10 & 5 & 5 & 5 \\
\hline \multicolumn{10}{|l|}{ Male } \\
\hline \multirow{4}{*}{ Bonemarrow } & Hematopoiesis, myeloid & 0 & $10^{\#}$ & $10^{\#}$ & $10^{\#}$ & $10^{\#}$ & 0 & $5^{+}$ & 0 \\
\hline & Minimal $(+1)$ & - & 0 & 0 & 0 & 0 & - & $5^{+}$ & 0 \\
\hline & Moderate $(+3)$ & - & 0 & 1 & 0 & 1 & - & 0 & 0 \\
\hline & Severe $(+4)$ & - & $10^{\#}$ & $9^{\#}$ & $10^{\#}$ & $9^{\#}$ & - & 0 & 0 \\
\hline \multirow{8}{*}{ Femorotibial joint } & Fibro-osseous lesion & 0 & $7^{+}$ & $5^{*}$ & $6^{*}$ & $8^{\#}$ & 0 & 0 & 1 \\
\hline & Minimal $(+1)$ & - & 4 & 3 & 4 & $5^{*}$ & - & - & 1 \\
\hline & Mild $(+2)$ & - & 3 & 1 & 2 & 3 & - & - & 0 \\
\hline & Moderate $(+3)$ & - & 0 & 1 & 0 & 0 & - & - & 0 \\
\hline & Degeneration, mucous, ligament & 0 & 0 & 0 & 1 & 0 & 0 & 0 & 0 \\
\hline & Minimal $(+1)$ & - & - & - & 1 & - & - & - & - \\
\hline & Marrow atrophy, focal & 0 & 0 & 0 & 0 & 0 & 0 & 0 & 1 \\
\hline & $\operatorname{Minimal}(+1)$ & - & - & - & - & - & - & - & 1 \\
\hline
\end{tabular}


Table 12. Continued

\begin{tabular}{|c|c|c|c|c|c|c|c|c|c|}
\hline \multirow{3}{*}{ Organs } & \multirow{2}{*}{$\begin{array}{c}\text { Groups } \\
\text { Findings }\end{array}$} & \multicolumn{5}{|c|}{ Main } & \multicolumn{3}{|c|}{ Recovery } \\
\hline & & G1 & G2 & G3 & G4 & G5 & G1 & G2 & G5 \\
\hline & Number examined & 10 & 10 & 10 & 10 & 10 & 5 & 5 & 5 \\
\hline \multicolumn{10}{|l|}{ Female } \\
\hline \multirow{6}{*}{ Bonemarrow } & Hematopoiesis, myeloid & 0 & $10^{\#}$ & $10^{\#}$ & $10^{\#}$ & $10^{\#}$ & 0 & 0 & 0 \\
\hline & Minimal $(+1)$ & - & 0 & 0 & 0 & 0 & - & - & - \\
\hline & Mild $(+2)$ & - & 0 & 0 & 0 & 0 & - & - & - \\
\hline & Moderate $(+3)$ & - & 0 & 0 & 1 & 0 & - & - & - \\
\hline & Severe $(+4)$ & - & $10^{\#}$ & $10^{\#}$ & $9^{\#}$ & $10^{\#}$ & - & - & - \\
\hline & Fibro-osseous lesion & 0 & 2 & 0 & 2 & 1 & 0 & 1 & 0 \\
\hline \multirow{12}{*}{ Femorotibial joint } & Minimal $(+1)$ & - & 2 & - & 1 & 0 & - & 1 & - \\
\hline & Mild $(+2)$ & - & 0 & - & 1 & 1 & - & 0 & - \\
\hline & Fibroplasia, synovial & 0 & 1 & 0 & 0 & 0 & 0 & 0 & 0 \\
\hline & Mild $(+2)$ & - & 1 & - & - & - & - & - & - \\
\hline & Hyperplasia, synovial cell & 0 & 3 & 0 & 2 & 0 & 0 & 1 & 1 \\
\hline & Minimal $(+1)$ & - & 0 & - & 1 & - & - & 1 & 0 \\
\hline & Mild $(+2)$ & - & 2 & - & 1 & - & - & 0 & 1 \\
\hline & Moderate $(+3)$ & - & 1 & - & 0 & - & - & 0 & 0 \\
\hline & Necrosis, synovial & 0 & 1 & 0 & 0 & 0 & 0 & 0 & 0 \\
\hline & Mild $(+2)$ & - & 1 & - & - & - & - & - & - \\
\hline & Serous atrophy, focal & 0 & 0 & 0 & 0 & 0 & 0 & 1 & 0 \\
\hline & $\operatorname{Minimal}(+1)$ & - & - & - & - & - & - & 1 & - \\
\hline
\end{tabular}

$-:$ No observed lesion in group. Statistically significant from G1 $\left({ }^{*} p<0.05 ;{ }^{+} p<0.01 ;{ }^{\sharp} p<0.00\right)$.

G1: Negative control, G2: Reference control, G3-G5: Test article treatment groups.

or $p<0.001)$. The main lesion observed on bone marrow (sternum) was due to the increase of myeloid hematopoiesis. It was significantly higher at all TS-DP2 doses and of the lenograstim group in both sexes $(p<0.001)$. The main lesion observed on the femorotibial joint was a fibro-osseous lesion, and other lesions were synovial cell hyperplasia and synovial cell necrosis. Fibro-osseous lesions were significantly higher at all TS-DP2 doses and of the lenograstim group in males $(p<0.05$ or $p<0.01$ or $p<0.001)$, and observed in 2, 2, and 1 case, respectively, of females in the lenograstim group and the at TS-DP2 doses of 500 and $1000 \mu \mathrm{g} / \mathrm{kg} /$ day. Synovial cell hyperplasia was observed of

Table 13. Histopathologic findings in the hindlimb and tarsus

\begin{tabular}{|c|c|c|c|c|c|c|c|c|c|}
\hline \multirow{3}{*}{ Organs } & \multirow{2}{*}{$\begin{array}{c}\text { Groups } \\
\text { Findings }\end{array}$} & \multicolumn{5}{|c|}{ Main } & \multicolumn{3}{|c|}{ Recovery } \\
\hline & & G1 & G2 & G3 & G4 & G5 & G1 & G2 & G5 \\
\hline & Number examined & 0 & 3 & 0 & 0 & 2 & 0 & 0 & 1 \\
\hline \multicolumn{10}{|l|}{ Male } \\
\hline \multirow{4}{*}{ Hindlimb } & Hematopoiesis, myeloid & & 2 & & & 1 & & & 0 \\
\hline & Severe $(+4)$ & & 2 & & & 1 & & & - \\
\hline & Fibro-osseous lesion & & 2 & & & 2 & & & 1 \\
\hline & Minimal $(+1)$ & & 0 & & & 0 & & & 1 \\
\hline \multirow{14}{*}{ Tarsus } & $\operatorname{Mild}(+2)$ & & 1 & & & 0 & & & 0 \\
\hline & Moderate $(+3)$ & & 1 & & & 0 & & & 0 \\
\hline & Severe $(+4)$ & & 0 & & & 2 & & & 0 \\
\hline & Edema, subcutis & & 2 & & & 2 & & & 1 \\
\hline & Moderate $(+3)$ & & 0 & & & 1 & & & 1 \\
\hline & Severe $(+4)$ & & 2 & & & 0 & & & 0 \\
\hline & Massive (+5) & & 0 & & & 1 & & & 0 \\
\hline & Hyperplasia, synovial cell & & 1 & & & 1 & & & 0 \\
\hline & Minimal $(+1)$ & & 0 & & & 0 & & & - \\
\hline & Mild(+2) & & 1 & & & 1 & & & - \\
\hline & Infiltration, inflammatory cell, connective tissue & & 2 & & & 2 & & & 1 \\
\hline & $\operatorname{Minimal}(+1)$ & & 0 & & & 0 & & & 0 \\
\hline & Mild(+2) & & 2 & & & 2 & & & 1 \\
\hline & Moderate $(+3)$ & & 0 & & & 0 & & & 0 \\
\hline
\end{tabular}


Table 13. Continued

\begin{tabular}{|c|c|c|c|c|c|c|c|c|c|}
\hline \multirow{3}{*}{ Organs } & \multirow{2}{*}{$\begin{array}{c}\text { Groups } \\
\text { Findings }\end{array}$} & \multicolumn{5}{|c|}{ Main } & \multicolumn{3}{|c|}{ Recovery } \\
\hline & & G1 & G2 & G3 & G4 & G5 & G1 & G2 & G5 \\
\hline & Number examined & 0 & 3 & 0 & 0 & 2 & 0 & 0 & 1 \\
\hline \multicolumn{10}{|l|}{ Female } \\
\hline \multirow{4}{*}{ Hindlimb } & Hematopoiesis, myeloid & & & & & 0 & & & \\
\hline & Severe $(+4)$ & & & & & - & & & \\
\hline & Fibro-osseous lesion & & & & & 1 & & & \\
\hline & $\operatorname{Minimal}(+1)$ & & & & & 0 & & & \\
\hline \multirow[t]{15}{*}{ Tarsus } & Mild $(+2)$ & & & & & 0 & & & \\
\hline & Moderate $(+3)$ & & & & & 0 & & & \\
\hline & Severe $(+4)$ & & & & & 1 & & & \\
\hline & Edema, subcutis & & & & & 1 & & & \\
\hline & Moderate $(+3)$ & & & & & 0 & & & \\
\hline & Severe $(+4)$ & & & & & 0 & & & \\
\hline & Massive $(+5)$ & & & & & 1 & & & \\
\hline & Hyperplasia, synovial cell & & & & & 1 & & & \\
\hline & Minimal $(+1)$ & & & & & 1 & & & \\
\hline & Mild(+2) & & & & & 0 & & & \\
\hline & Infiltration, inflammatory cell, connective tissue & & & & & 1 & & & \\
\hline & $\operatorname{Minimal}(+1)$ & & & & & 0 & & & \\
\hline & Mild $(+2)$ & & & & & 1 & & & \\
\hline & Necrosis, synovial cell & & & & & 1 & & & \\
\hline & Minimal $(+1)$ & & & & & 1 & & & \\
\hline
\end{tabular}

-: No observed lesion in group. Statistically significant from G1 $\left({ }^{*} p<0.05 ;{ }^{+} p<0.01 ;{ }^{\sharp} p<0.00\right)$.

G1: Negative control, G2: Reference control, G3-G5: Test article treatment groups.

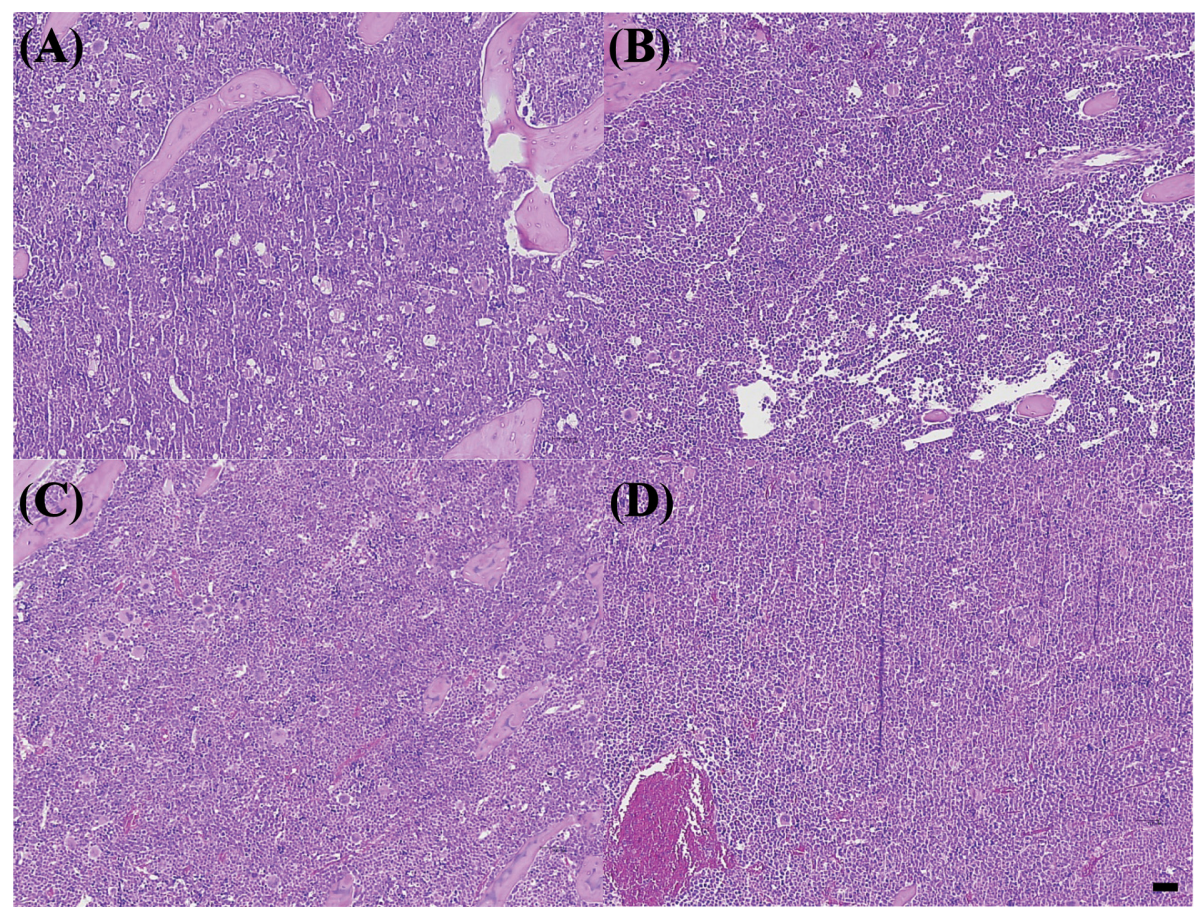

Fig. 4. Myeloid hematopoeisis of the bone marrow in Sprague-Dawley rats treated with TS-DP2 and Lenograstim (postive control) $1000 \mu \mathrm{g} / \mathrm{kg} /$ day for 4 weeks. H\&E stain, Animal No. 16 (A) No. 51 (B) No. 82 (C) No. 119 (D) Bar $=100 \mu \mathrm{m}$.

3 and 2 cases in females of the lenograstim group and the TS-DP2 dose of $500 \mu \mathrm{g} / \mathrm{kg} / \mathrm{day}$, respectively. Synovial cell necrosis was observed only in females in the lenograstim group.

In animals with severe hind limb edema observed on necropsy, myeloid hematopoiesis was observed in 2 and 1 case, 


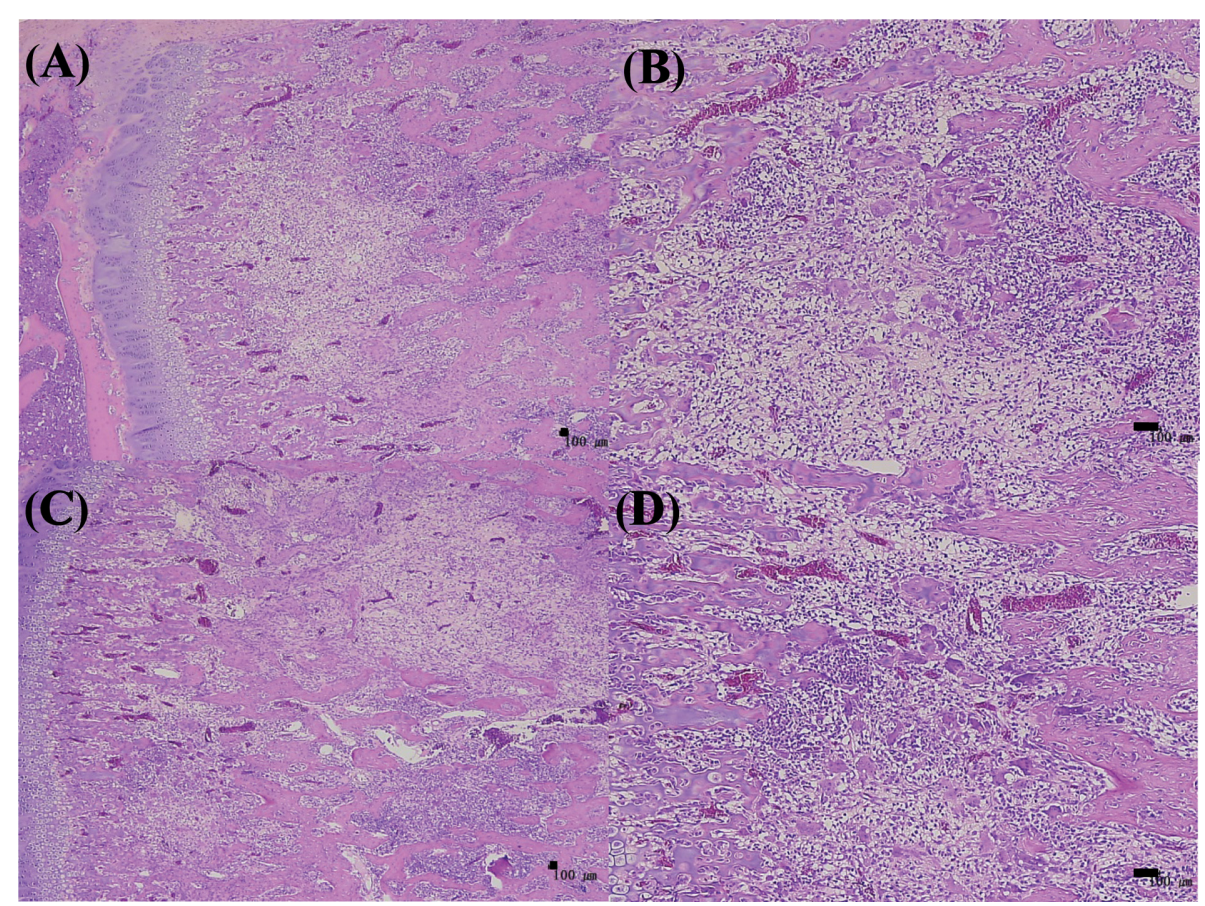

Fig. 5. Fibro-osseous lesion of the femorotibial joint in a male Sprague-Dawley rats treated with TS-DP2 and Lenograstim (postive control) $1000 \mu \mathrm{g} / \mathrm{kg} /$ day for 4 weeks. H\&E stain, Animal No. 17 (A,B), No. 56 (C,D), Bar = $100 \mu \mathrm{m}$.

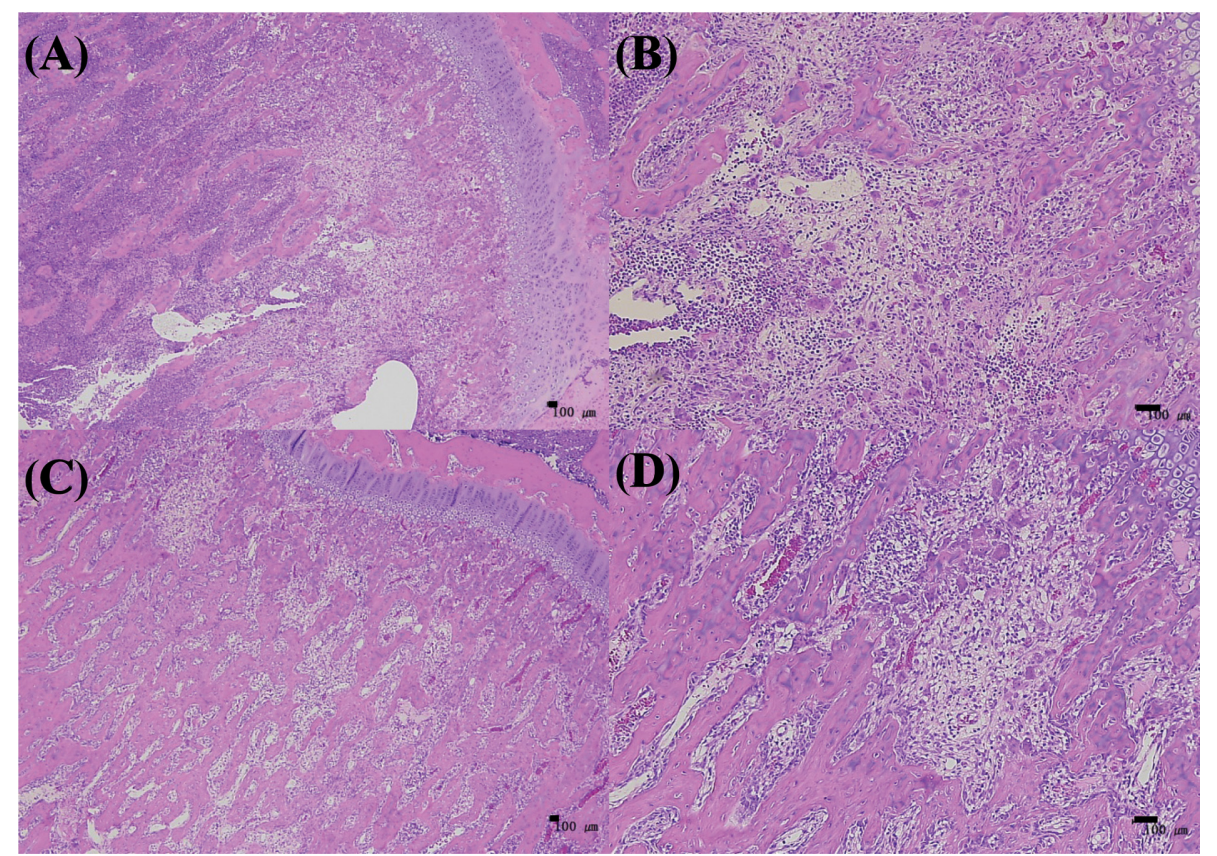

Fig. 6. Fibro-osseous lesion of the femorotibial joint in a female Sprague-Dawley rats treated with TS-DP2 and Lenograstim (postive control) $1000 \mu \mathrm{g} / \mathrm{kg} /$ day for 4 weeks. H\&E stain, Animal No. 82 (A, B), No. 123 (C, D), Bar = $100 \mu \mathrm{m}$.

respectively, of males of the lenograstim group and the TSDP2 group at a dose of $1000 \mu \mathrm{g} / \mathrm{kg} /$ day. The tarsus region of the hind limb part had sporadically observed fibro-osseous lesion, edema, inflammatory cell infiltration on connective tissue, and synovial cell necrosis. Other organs demonstrated similar findings on histopathological examination, but except increased size and dark red discoloration of the spleen observed on 1 female receiving $250 \mu \mathrm{g} / \mathrm{kg} /$ day TS- 
DP2, any abnormal lesion was not detected.

In the recovery group, myeloid extramedullary hematopoiesis and infiltration of mononuclear cell were significantly increased at a dose of $1000 \mu \mathrm{g} / \mathrm{kg} /$ day in males $(p<$ $0.05)$. The myeloid hematopoiesis observed in bone marrow was significantly higher in the lenograstim group males $(p<0.01)$. The fibro-osseous lesion observed on the femorotibial joint was observed in 1 case at the TS-DP2 dose of
$1000 \mu \mathrm{g} / \mathrm{kg} /$ day in males and in the lenograstim group females, and synovial cell hyperplasia was observed in 1 case in the lenograstim group and in 1 case in the TS-DP2 $1000 \mu \mathrm{g} / \mathrm{kg} /$ day dose in females. Serous atrophy occurred in 1 case in the lenograstim group in females. Other nonspecific lesions were considered to occur spontaneously in SD rats $(9,10)$.

Histopathological examination resulted in similar obser-

Table 14. Toxicokinetic parameters intravenously treated with TS-DP2 for 4 weeks

\begin{tabular}{|c|c|c|c|c|c|}
\hline Groups & Doses $(\mu \mathrm{g} / \mathrm{kg} /$ day $)$ & Duration & Sexes & $\mathrm{AUC}_{0-24 \mathrm{~h}}(\mathrm{hr} * \mathrm{ng} / \mathrm{mL})$ & $\mathrm{C}_{0}(\mathrm{ng} / \mathrm{mL})$ \\
\hline \multirow{4}{*}{$\mathrm{G} 2$} & \multirow{4}{*}{1000} & \multirow{2}{*}{ Day 1} & Male & 17311.7 & 13449.6 \\
\hline & & & Female & 16091.2 & 13881.9 \\
\hline & & \multirow{2}{*}{ Day 28} & Male & 20174.1 & 12390.7 \\
\hline & & & Female & 12003.6 & 10141.5 \\
\hline \multirow{4}{*}{ G3 } & \multirow{4}{*}{250} & \multirow{2}{*}{ Day 1} & Male & 3310.5 & 2504.4 \\
\hline & & & Female & 2693.0 & 2502.7 \\
\hline & & \multirow{2}{*}{ Day 28} & Male & 5593.0 & 4122.0 \\
\hline & & & Female & 3673.2 & 4434.9 \\
\hline \multirow{4}{*}{ G4 } & \multirow{4}{*}{500} & \multirow{2}{*}{ Day 1} & Male & 7920.8 & 6425.9 \\
\hline & & & Female & 7361.9 & 6254.2 \\
\hline & & \multirow{2}{*}{ Day 28} & Male & 10138.0 & 8434.7 \\
\hline & & & Female & 6927.4 & 6867.5 \\
\hline \multirow{4}{*}{ G5 } & \multirow{4}{*}{1000} & \multirow{2}{*}{ Day 1} & Male & 16183.3 & 12222.2 \\
\hline & & & Female & 14899.7 & 11768.9 \\
\hline & & \multirow{2}{*}{ Day 28} & Male & 21291.0 & 18216.7 \\
\hline & & & Female & 19573.1 & 17886.4 \\
\hline \multicolumn{6}{|c|}{ Grand mean (male and female average) } \\
\hline \multirow{2}{*}{ G2 } & \multirow{2}{*}{1000} & Day 1 & & 16701.5 & 13665.7 \\
\hline & & Day 28 & & 20432.0 & 18051.6 \\
\hline \multirow{2}{*}{ G3 } & \multirow{2}{*}{250} & Day 1 & & 3001.7 & 2503.5 \\
\hline & & Day 28 & & 4633.1 & 4278.4 \\
\hline \multirow{2}{*}{ G4 } & \multirow{2}{*}{500} & Day 1 & & 7641.4 & 6340.0 \\
\hline & & Day 28 & & 8532.7 & 7651.1 \\
\hline \multirow{2}{*}{ G5 } & \multirow{2}{*}{1000} & Day 1 & & 15541.5 & 11995.5 \\
\hline & & Day 28 & & 16088.9 & 11266.1 \\
\hline
\end{tabular}

Table 15. Serum concentrations of G-CSF intravenously treated with TS-DP2 for 4 weeks on Day 1

\begin{tabular}{|c|c|c|c|c|}
\hline \multirow{2}{*}{ Groups } & \multirow{2}{*}{$\begin{array}{c}\text { Doses } \\
(\mu \mathrm{g} / \mathrm{kg} / \text { day })\end{array}$} & \multirow{2}{*}{$\begin{array}{l}\text { Time } \\
(\mathrm{hr})\end{array}$} & \multicolumn{2}{|c|}{ Concentrations (ng/mL) } \\
\hline & & & Male & Female \\
\hline \multirow[t]{8}{*}{$\mathrm{G} 2$} & 1000 & 0 & $0.0 \pm 0.0$ & $0.0 \pm 0.0$ \\
\hline & & 0.08 & $12120.6 \pm 710.2$ & $12474.6 \pm 853.5$ \\
\hline & & 0.5 & $7204.5 \pm 194.9$ & $7309.9 \pm 256.6$ \\
\hline & & 1 & $3700.6 \pm 411.2$ & $3603.1 \pm 225.7$ \\
\hline & & 2 & $2000.1 \pm 443.4$ & $1685.6 \pm 258.6$ \\
\hline & & 4 & $816.1 \pm 35.5$ & $781.8 \pm 27.0$ \\
\hline & & 8 & $219.4 \pm 41.9$ & $146.7 \pm 19.3$ \\
\hline & & 24 & $0.2 \pm 0.2$ & $0.1 \pm 0.1$ \\
\hline \multirow[t]{8}{*}{ G3 } & 250 & 0 & $0.0 \pm 0.0$ & $0.0 \pm 0.0$ \\
\hline & & 0.08 & $2347.4 \pm 706.5$ & $2240.4 \pm 303.3$ \\
\hline & & 0.5 & $698.3 \pm 59.8$ & $1288.0 \pm 28.2$ \\
\hline & & 1 & $751.8 \pm 157.2$ & $655.8 \pm 56.9$ \\
\hline & & 2 & $366.9 \pm 107.1$ & $262.7 \pm 16.7$ \\
\hline & & 4 & $154.4 \pm 17.9$ & $117.4 \pm 11.9$ \\
\hline & & 8 & $26.4 \pm 3.4$ & $20.0 \pm 6.3$ \\
\hline & & 24 & $0.0 \pm 0.0$ & $0.0 \pm 0.0$ \\
\hline
\end{tabular}


Table 15. Continued

\begin{tabular}{|c|c|c|c|c|}
\hline \multirow{2}{*}{ Groups } & \multirow{2}{*}{$\begin{array}{c}\text { Doses } \\
(\mu \mathrm{g} / \mathrm{kg} / \mathrm{day})\end{array}$} & \multirow{2}{*}{$\begin{array}{l}\text { Time } \\
(\mathrm{hr})\end{array}$} & \multicolumn{2}{|c|}{ Concentrations (ng/mL) } \\
\hline & & & Male & Female \\
\hline \multirow[t]{8}{*}{ G4 } & 500 & 0 & $0.0 \pm 0.0$ & $0.0 \pm 0.0$ \\
\hline & & 0.08 & $5758.4 \pm 347.5$ & $5698.3 \pm 1019.7$ \\
\hline & & 0.5 & $3327.9 \pm 328.5$ & $3577.8 \pm 125.3$ \\
\hline & & 1 & $1849.6 \pm 117.5$ & $1455.5 \pm 226.1$ \\
\hline & & 2 & $871.0 \pm 115.6$ & $898.8 \pm 145.3$ \\
\hline & & 4 & $395.6 \pm 81.4$ & $337.2 \pm 12.4$ \\
\hline & & 8 & $80.8 \pm 9.4$ & $58.5 \pm 1.8$ \\
\hline & & 24 & $0.0 \pm 0.0$ & $0.0 \pm 0.0$ \\
\hline \multirow[t]{8}{*}{ G5 } & 1000 & 0 & $0.0 \pm 0.0$ & $0.0 \pm 0.0$ \\
\hline & & 0.08 & $11113.4 \pm 513.4$ & $10708.8 \pm 795.5$ \\
\hline & & 0.5 & $6907.9 \pm 213.4$ & $6679.8 \pm 435.8$ \\
\hline & & 1 & $3403.5 \pm 418.2$ & $3793.0 \pm 337.5$ \\
\hline & & 2 & $855.3 \pm 43.4$ & $1447.0 \pm 430.9$ \\
\hline & & 4 & $775.5 \pm 126.7$ & $798.7 \pm 137.1$ \\
\hline & & 8 & $206.5 \pm 2.5$ & $125.8 \pm 15.7$ \\
\hline & & 24 & $0.3 \pm 0.2$ & $0.1 \pm 0.2$ \\
\hline
\end{tabular}

Each value represents mean $\pm S D(n=6)$.

Table 16. Serum concentrations of G-CSF intravenously treated with TS-DP2 for 4 weeks on Day 28

\begin{tabular}{|c|c|c|c|c|}
\hline \multirow{2}{*}{ Groups } & \multirow{2}{*}{$\begin{array}{c}\text { Doses } \\
(\mu \mathrm{g} / \mathrm{kg} / \mathrm{day})\end{array}$} & \multirow{2}{*}{$\begin{array}{l}\text { Time } \\
(\mathrm{hr})\end{array}$} & \multicolumn{2}{|c|}{ Concentrations (ng/mL) } \\
\hline & & & Male & Female \\
\hline \multirow[t]{8}{*}{ G2 } & 1000 & 0 & $0.2 \pm 0.2$ & $0.2 \pm 0.2$ \\
\hline & & 0.08 & $11562.4 \pm 579.6$ & $9189.4 \pm 1049.0$ \\
\hline & & 0.5 & $8180.7 \pm 1401.8$ & $5613.3 \pm 634.6$ \\
\hline & & 1 & $5272.2 \pm 894.2$ & $2941.2 \pm 348.0$ \\
\hline & & 2 & $2653.7 \pm 434.3$ & $1342.0 \pm 155.6$ \\
\hline & & 4 & $1108.7 \pm 113.1$ & $595.8 \pm 92.0$ \\
\hline & & 8 & $175.5 \pm 39.7$ & $70.3 \pm 3.1$ \\
\hline & & 24 & $0.2 \pm 0.2$ & $0.1 \pm 0.1$ \\
\hline \multirow[t]{8}{*}{ G3 } & 250 & 0 & $0.2 \pm 0.1$ & $0.0 \pm 0.0$ \\
\hline & & 0.08 & $3742.6 \pm 252.1$ & $3667.5 \pm 379.7$ \\
\hline & & 0.5 & $2309.4 \pm 143.8$ & $1418.5 \pm 629.1$ \\
\hline & & 1 & $1533.1 \pm 172.6$ & $965.8 \pm 265.4$ \\
\hline & & 2 & $697.4 \pm 44.0$ & $418.0 \pm 51.1$ \\
\hline & & 4 & $309.2 \pm 36.7$ & $158.3 \pm 10.5$ \\
\hline & & 8 & $30.3 \pm 2.6$ & $9.4 \pm 4.8$ \\
\hline & & 24 & $0.1 \pm 0.2$ & $0.1 \pm 0.2$ \\
\hline \multirow[t]{8}{*}{ G4 } & 500 & 0 & $0.1 \pm 0.1$ & $0.1 \pm 0.1$ \\
\hline & & 0.08 & $7445.7 \pm 820.1$ & $6083.2 \pm 162.3$ \\
\hline & & 0.5 & $3991.1 \pm 1086.9$ & $3317.4 \pm 502.2$ \\
\hline & & 1 & $2647.8 \pm 113.9$ & $1741.4 \pm 114.5$ \\
\hline & & 2 & $1263.9 \pm 30.8$ & $670.2 \pm 185.2$ \\
\hline & & 4 & $532.7 \pm 38.7$ & $309.0 \pm 19.4$ \\
\hline & & 8 & $61.6 \pm 22.5$ & $36.1 \pm 4.8$ \\
\hline & & 24 & $0.0 \pm 0.0$ & $0.0 \pm 0.0$ \\
\hline \multirow[t]{8}{*}{ G5 } & 1000 & 0 & $0.4 \pm 0.1$ & $0.4 \pm 0.1$ \\
\hline & & 0.08 & $15285.7 \pm 1200.6$ & $15941.6 \pm 3032.9$ \\
\hline & & 0.5 & $6358.5 \pm 1976.5$ & $8965.6 \pm 214.5$ \\
\hline & & 1 & $5372.7 \pm 171.1$ & $5056.8 \pm 301.8$ \\
\hline & & 2 & $2722.1 \pm 196.7$ & $1861.4 \pm 124.6$ \\
\hline & & 4 & $1135.4 \pm 33.2$ & $994.6 \pm 181.2$ \\
\hline & & 8 & $227.5 \pm 8.9$ & $116.5 \pm 21.5$ \\
\hline & & 24 & $0.3 \pm 0.1$ & $0.0 \pm 0.0$ \\
\hline
\end{tabular}

Each value represents mean $\pm S D(n=6)$ 
vances between the lenograstim group and the TS-DP2 $1000 \mu \mathrm{g} / \mathrm{kg} /$ day group, in both degree and occurrence of lesions.

Toxicokinetics study. The toxicokinetic parameters of G-CSF are displayed in Table 14. The serum concentrations are displayed in Table 15 and 16, and graphically in Fig. 7 and 8. The negative control (G0) (Water for injection), positive reference article (G2) (Choongwae Neutrogin Injection: $1000 \mu \mathrm{g} / \mathrm{kg} / \mathrm{day}$ ), and test article (TS-DP2: 250 [G3], 500 [G4], and 1000 [G5] $\mu \mathrm{g} / \mathrm{kg} /$ day) were intravenously administered to male and female rats for 4 weeks.

On administration initiation day (Day 1), the G-CSF in rat serum remained at a concentration above LLOQ (0.2 ng/ $\mathrm{mL})$ until $8 \mathrm{hr}$ after administration in G3 $(250 \mu \mathrm{g} / \mathrm{kg} /$ day $)$ and G4 $(500 \mu \mathrm{g} / \mathrm{kg} /$ day $)$. In some animals in the G2 (1000 $\mu \mathrm{g} / \mathrm{kg} /$ day) and G5 (1000 $\mu \mathrm{g} / \mathrm{kg} /$ day $)$ groups, the concentration was above the LLOQ until the last blood sampling time $(24 \mathrm{hr})$. Overall, G-CSF in rat serum persisted until at least $8 \mathrm{hr}$ after administration. On Day 28, the G-CSF in rat serum was measured at above the LLOQ at prior administration, and until the last blood sampling time in some animals. Overall, the G-CSF in rat serum was maintained until

(A)

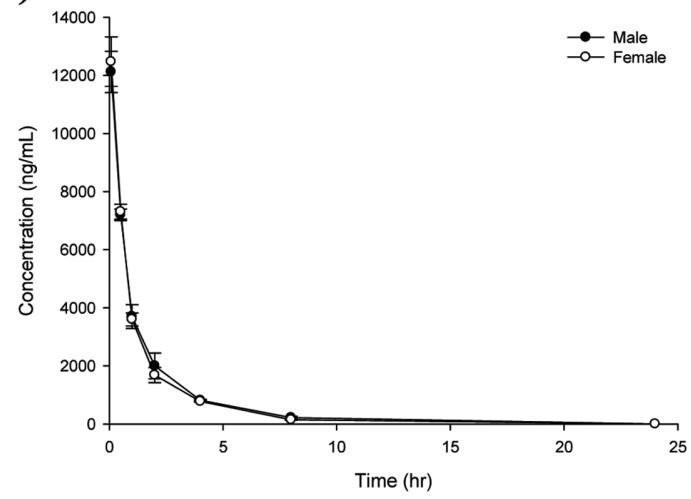

(C)

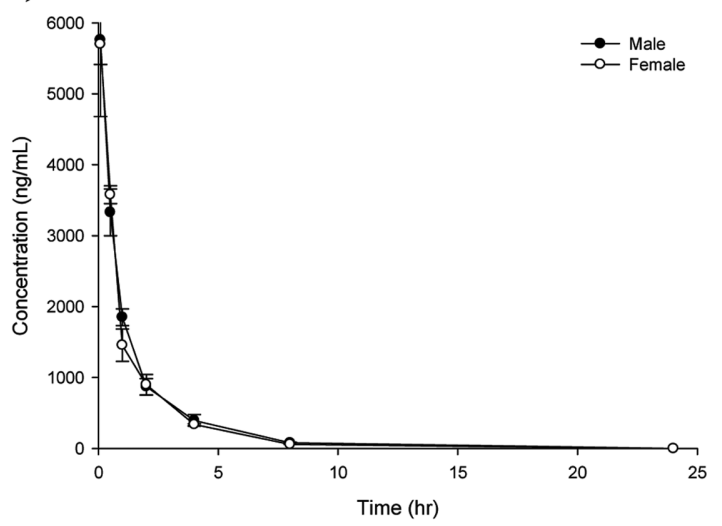

$8 \mathrm{hr}$ after administration on Day 1 and Day 28. G-CSF was not detected in the negative control group.

On Day 1, the ratios of male to female of $\mathrm{AUC}_{0-24 \mathrm{~h}}$ and $\mathrm{C}_{0}$ values were 1.1- and 1.0-fold (G2), 1.2- and 1.0-fold (G3), 1.1- and 1.0-fold (G4), and 1.1- and 1.0-fold (G5), and on Day 28, 1.7- and 1.2-fold (G2), 1.5- and 0.9-fold (G3), 1.5- and 1.2-fold (G4), and 1.1- and 1.1-fold (G5), respectively. The systemic exposure $\left(\mathrm{AUC}_{0-24 \mathrm{~h}}\right.$ and $\left.\mathrm{C}_{0}\right)$ was not markedly different $(>2$-fold or $<0.5$-fold) between male and female rats.

When the doses were gradually increased 2.0 -fold, the grand means of $\mathrm{AUC}_{0-24 \mathrm{~h}}$ values were increased 2.5- and 2.0-fold (Day 1) or 1.8- and 1.9-fold (Day 28), and the grand means of $\mathrm{C}_{0}$ values were increased 2.5- and 1.9-fold (Day 1) or 1.8- and 1.5-fold (Day 28). Overall, the systemic exposure $\left(\mathrm{AUC}_{0-24 \mathrm{~h}}\right.$ and $\left.\mathrm{C}_{0}\right)$ increased in a doserelated manner.

The ratios of Day 28 to Day 1 of grand mean $\mathrm{AUC}_{0-24 \mathrm{~h}}$ and $\mathrm{C}_{0}$ values were 1.2- and 1.3-fold (G2), 1.5- and 1.7-fold (G3), 1.1- and 1.2-fold (G4), and 1.0- and 0.9-fold (G5), respectively. Therefore the systemic exposure $\left(\mathrm{AUC}_{0-24 \mathrm{~h}}\right.$ and $\mathrm{C}_{0}$ ) was not markedly different $(>2$-fold or $<0.5$-fold) in the administration period.
(B)

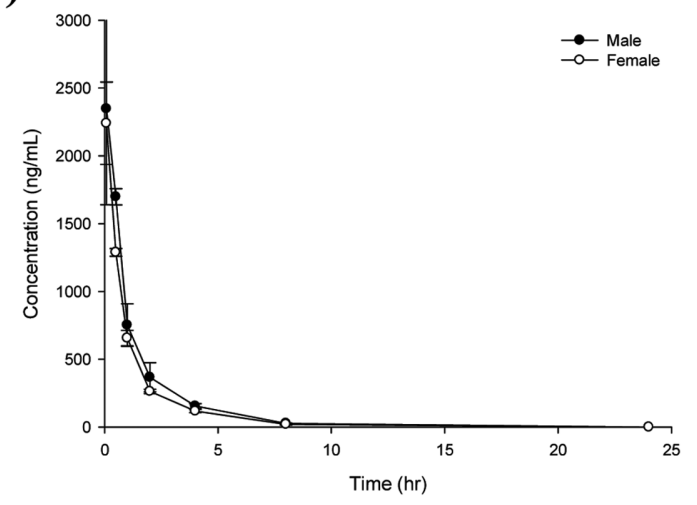

(D)

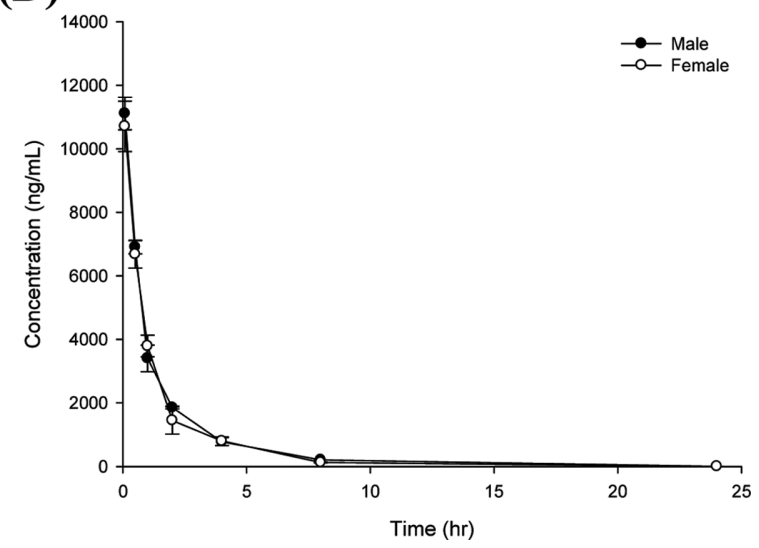

Fig. 7. Mean serum concentration-time profiles of G-CSF at G2 (1000 $\mu \mathrm{g} / \mathrm{kg} / \mathrm{day}, \mathrm{A}), \mathrm{G} 3(250 \mu \mathrm{g} / \mathrm{kg} / \mathrm{day}, \mathrm{B}), \mathrm{G} 4(500 \mu \mathrm{g} / \mathrm{kg} / \mathrm{day}, \mathrm{C})$, and G5 $(1000 \mu \mathrm{g} / \mathrm{kg} /$ day, D) on Day 1 . 
(A)

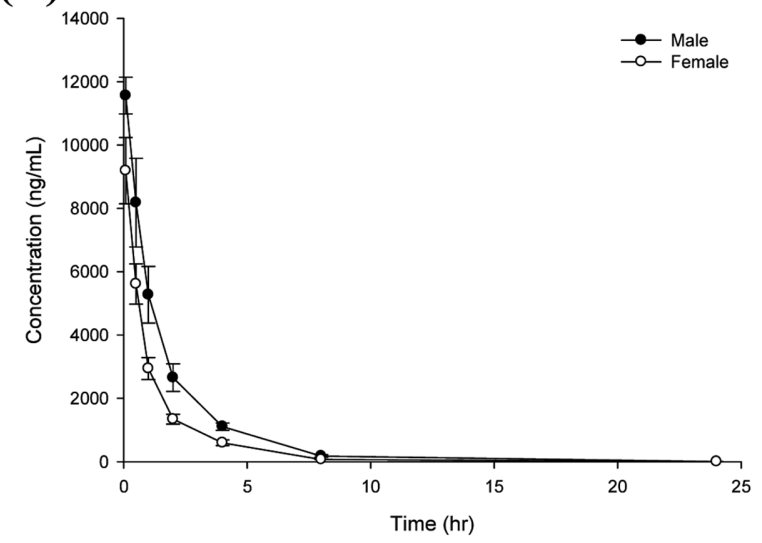

(C)

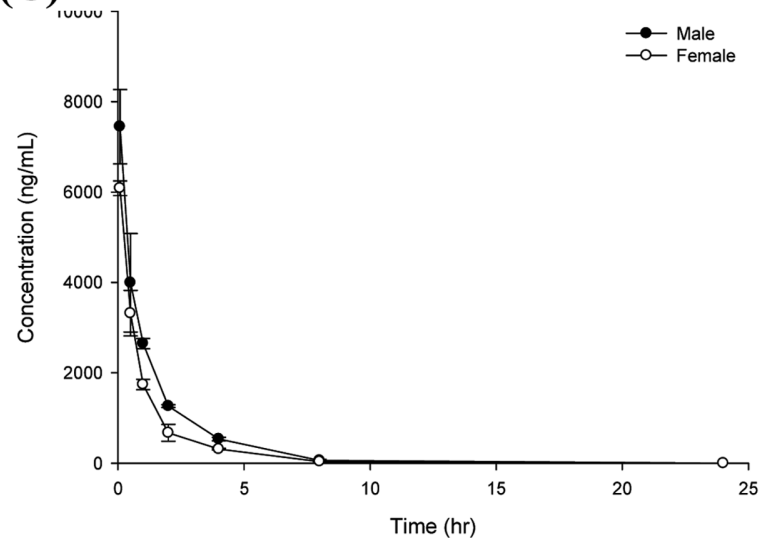

(B)

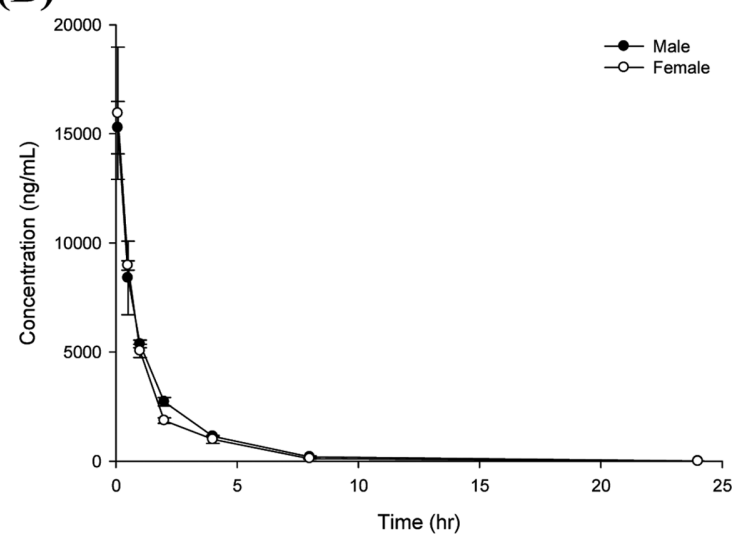

(D)

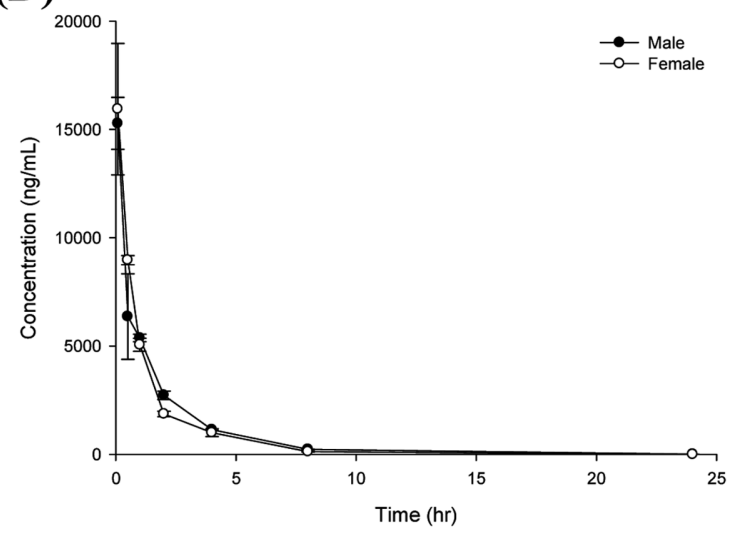

Fig. 8. Mean serum concentration-time profiles of G-CSF at G2 $(1000 \mu \mathrm{g} / \mathrm{kg} / \mathrm{day}, \mathrm{A}), \mathrm{G} 3(250 \mu \mathrm{g} / \mathrm{kg} / \mathrm{day}, \mathrm{B}), \mathrm{G} 4(500 \mu \mathrm{g} / \mathrm{kg} / \mathrm{day}, \mathrm{C})$, and G5 $(1000 \mu \mathrm{g} / \mathrm{kg} /$ day, D) on Day 28.

The ratios of grand mean $\mathrm{AUC}_{0-24 \mathrm{~h}}$ and $\mathrm{C}_{0}$ values of TSDP2 to lenograstim were 0.9 - and 0.9-fold (Day 1) and 0.8and 0.6 -fold (Day 28$)$ at the same dose $(1000 \mu \mathrm{g} / \mathrm{kg} /$ day), respectively. Therefore, the systemic exposure of TS-DP2 was similar to that of lenograstim.

In conclusion, systemic exposure of G-CSFs tested was not markedly different between males and females, and exhibited non-linear kinetics and accumulation at all dose levels. Therefore, the toxicokinetic profile of TS-DP2 is similar to that of lenograstim.

\section{DISCUSSION}

We conducted toxicity and toxicokinetic studies in SD rats to compare TS-DP2, a new rhG-CSF, and lenograstim, a reference rhG-CSF. The toxicity test was conducted over 4 weeks using IV injection and allowing for a 2-week recovery period. The toxicokinetic test was done separately from the toxicity test. Most of the results in these studies were similar to previous reports for G-CSF products.

In a 4-week repeated dose intravenous toxicity study, there was no significant difference between TS-DP2 and lenograstim in the subjects' water consumption, ophthalmological examination, and urinalysis. Clinically, hind limb edema, abnormal gait, and ataxia were observed in the TSDP2 $1000 \mu \mathrm{g} / \mathrm{kg} /$ day group, and hind limb edema was observed in the lenograstim $1000 \mu \mathrm{g} / \mathrm{kg} /$ day group. These observations were considered effects of the G-CSF, and were improved in the recovery period. A decrease in mean body weight or body weight gain was observed in male main and recovery groups receiving lenograstim, as well as dose of $1000 \mu \mathrm{g} / \mathrm{kg} / \mathrm{day}$. There were no significant differences between the TS-DP2 group and the lenograstim group. RBC decreased in both sexes in the lenograstim group and male main groups at all doses, and in females in the TS-DP2 group. Increase in RDW was considered dosedependent, and changes in erythroid extramedullary hematopoiesis in the spleen and liver were observed. These changes seemed to be related to treatment with G-CSF, but were considered not to be indicative of toxicology, as the numerical values measured were within range or only slightly elevated compared to the normal reference range provided by Chemon Inc. Significant increases in WBC observed in both sexes at all TS-DP2 doses and in the 
lenograstim group were considered to be related to an increase in neutrophils (11), myeloid extramedullary hematopoiesis in spleen and liver, and myeloid hematopoiesis on bone marrow. This observation is expected, considering the pharmacological action of a G-CSF medication. The changes were within the normal reference range of SD rats in the recovery groups, so they were considered non-toxicological changes. The significant decrease of PLT observed in males at all doses of TS-DP2 and in both sexes in the lenograstim group was attributed to the G-CSF, and similar to the increased MPV and the decreased in PLT on females. In a previous study, PLT were decreased after lenograstim administration (12). These changes were considered nontoxicological changes.

In a clinical biochemistry test, the significant increase of ALP observed in both sexes in the lenograstim group and at all doses of TS-DP2 was considered to be related to the GCSF. It was also related to the formation of fibro-osseous lesion on the femorotibial joint and tartus. This was related with the increase in neutrophil count, as ALP was a component within the neutrophil $(13,14)$. ALP was likewise increased after lenograstim administration (14), which was attributed to pharmacological activity by G-CSF. The changes in clinical biochemistry items observed at the reference control and test article recovery groups were considered to be unrelated to the respective medications, because they were not observed in the main groups or observed only in a single sex, were within normal reference range for SD rats, or the variance was small.

The significant increase of absolute and relative of spleen organ weight was observed dose-dependently in both sexes at all doses of TS-DP2 and the lenograstim group was considered to be related to the pharmacological effect of GCSF. It was also related to the increased size of the spleen in necropsy findings, and the increase of erythroid and myeloid extramedullary hematopoiesis in the spleen seen on histopathological examination. This was considered to undergo delayed recovery, but did not cause abnormal lesions on histopathological examination. Therefore, they were considered non-toxicological changes. The significant decrease in fasting body weight in the main and recovery groups of lenograstim in males was considered to be related to the drug, which showed a significant suppression of weight gain (approximately 10\%). Other changes were considered spontaneous, because they were not observed on hematological tests, clinical biochemistry tests, or necropsy findings.

In necropsy finding, the hind limb edema in both sexes in the lenograstim group and at all doses in the TS-DP2 group was attributed to the pharmacological activity of G-CSF. It was also related to the myeloid hematopoiesis and fibroosseous lesions observed on the hind limb (contained tartus) upon histopathological examination.

The lesions observed on histopathological examination on the spleen, femur, sternum, and hind limb (contained tar- tus) exhibited a dose-dependent relationship. The lesions were considered to be related to the G-CSF given, and were observed on hematological and clinical biochemistry tests. Among them, the myeloid extramedullary hematopoiesis on liver and spleen-related myeloid hematopoiesis on bone marrow was considered a pharmacological action of the GCSF. The fibro-osseous lesion on the femorotibial joint characterized by increased appearance of osteoclasts and fibroblasts, and osteoanagenesis was observed in a part of femorotibial joint. The increased number of osteoclasts was attributed to the pharmacological activity of TS-DP2 and lenograstim. It was observed in both sexes in the lenograstim group, at all TS-DP2 doses in males, and at the TS-DP2 dose of $500 \mu \mathrm{g} / \mathrm{kg} /$ day or more in females. They were observed changes related to the lesions on histopathology examination, similar to hind limb edema and ataxia. Fibroosseous lesions were considered to be toxicological changes, and were observed in both sexes in the lenograstim group, at all TS-DP2 doses in males, and at the TS-DP2 dose of $500 \mu \mathrm{g} / \mathrm{kg} /$ day or more in females. Osteolysis or osteogenesis were expected, as well as weakening of the bone and the subsequent effects on surrounding tissues, including as blood vessels, nerves, and bone marrow. The infiltration of mononuclear cells in the liver and the increase of erythroid extramedullary hematopoiesis in the spleen and liver were observed in both sexes in the lenograstim group and at all doses of TS-DP2. This infiltration effect was attributed to the pharmacological activity of a G-CSF, because related changes were observed on hematological tests. It was considered to be a non-toxicological change, because the data were within the reference range or only slightly over and improved in the recovery group. Other lesions that have previously been shown to occur spontaneously in $\mathrm{SD}$ rats were considered to be unrelated to the test article $(9,10,15-19)$.

In a 4-week repeated IV dose study of TS-DP2 in SD rats, including a 2-week recovery period, there were several histological, biochemical, and physiological changes attributed to the G-CSF. Clinical hind limb edema, the decrease of mean and gain in body weight in males in the lenograstim and high-dose TS-DP2 group, the decrease of RBC and PLT, the increase of items related to WBC on hematological tests, the increase of ALP on biochemistry tests, the increase of the absolute and relative weights of spleen, and the lesions on liver, spleen, femur, sternum, hind limb, and tartus on histopathological examination were considered to be related to the reference control and test article. Above all, the fibro-osseous lesion observed on both sexes in the lenograstim group, at all TS-DP2 doses in males, and at TS-DP2 dose of $500 \mu \mathrm{g} / \mathrm{kg} /$ day or more in females was considered to be a toxicological change.

In conclusion, the toxicological target of TS-DP2 is primarily bone, with a lowest observed adverse effect level (LOAEL) in males set at $250 \mu \mathrm{g} / \mathrm{kg} / \mathrm{day}$, and no observed adverse effect level (NOAEL) in females at $250 \mu \mathrm{g} / \mathrm{kg} /$ day 
in this study.

The TS-DP2 dose of $1000 \mu \mathrm{g} / \mathrm{kg} /$ day has shown similar effects to lenograstim at a dose of $1000 \mu \mathrm{g} / \mathrm{kg} /$ day in this study under these experimental conditions. In the toxicokinetic study, the ratios (lenograstim versus TS-DP2) of grand mean $\mathrm{AUC}_{0-24 \mathrm{~h}}$ and $\mathrm{C}_{0}$ values were 0.9- and 0.9-fold (Day 1) and 0.8- and 0.6-fold (Day 28) at the same dose level $(1000 \mu \mathrm{g} / \mathrm{kg} / \mathrm{day})$. The systemic exposure of TS-DP2 was similar to lenograstim. Therefore, the systemic exposure of the two G-CSF products was similar in males and females, showed non-linear kinetics, and was able to accumulate in all dose levels. The systemic exposure of TS-DP2 was similar to lenograstim at the same dose.

Most of the results were attributed to the pharmacological action of rhG-CSF. Although there were some differences observed in the toxicity tests, they were not thought to represent a significant discrepancy between TS-DP2 and lenograstim. Consequently, the test article TS-DP2 was shown to be biologically similar to lenograstim in rats.

\section{REFERENCES}

1. Glaspy, J.A. (2003) Hematopoietic management in oncology practice. Part 1. Myeloid growth factors. Oncology, 17, 15931603.

2. El Ouriaghli, F., Fujiwara, H., Melenhorst, J.J., Sconocchia, G., Hensel, N. and Barrett, A.J. (2003) Neutrophil elastase enzymatically antagonizes the in vitro action of G-CSF: implications for the regulation of granulopoiesis. Blood, 101, 17521758.

3. Yong, K.L. (1996) Granulocyte colony-stimulating factor (G$\mathrm{CSF}$ ) increases neutrophil migration across vascular endothelium independent of an effect on adhesion: Comparison with granulocyte-macrophage colony-stimulating factor (GMCSF). Br. J. Haematol., 94, 40-47.

4. Morstyn, G., Campbell, L., Souza, L.M., Alton, N.K., Keech, J., Green, M., Sheridan, W., Metcalf, D. and Fox, R. (1988) Effect of granulocyte colony stimulating factor on neutropenia induced by cytotoxic chemotherapy. Lancet, 1, 667-672.

5. Price, T.H., Chatta, G.S. and Dale, D.C. (1996) Effect of recombinant granulocyte colony-stimulating factor on neutrophil kinetics in normal young and elderly humans. Blood, $\mathbf{8 8}$, 335-340.

6. Glaspy, J.A., Baldwin, G.C., Robertson, P.A., Souza, L., Vincent, M., Ambersley, J. and Golde, D.W. (1988) Therapy for neutropenia in hairy cell leukemia with recombinant human granulocyte colony-stimulating factor. Ann. Intern. Med., 109, 789-795.

7. Yokose, N., Ogata, K., Tamura, H., An, E., Nakamura, K.,
Kamikubo, K., Kudoh, S., Dan, K. and Nomura, T. (1998) Pulmonary toxicity after granulocyte colonystimulating factor-combined chemotherapy for non-Hodgkin's lymphoma. Br. J. Cancer, 77, 2286-2290.

8. Johnston, E., Crawford, J., Blackwell, S., Bjurstrom, T., Lockbaum, P., Roskos, L., Yang, B.B., Gardner, S., Miller-Messana, M.A., Shoemaker, D., Garst, J. and Schwab, G. (2000) Randomized, dose-escalation study of SD/ 01 compared with daily filgrastim in patients receiving chemotherapy. J. Clin. Oncol., 18, 2522-2528.

9. Inazawa, T. (2014) A case of platelet and white blood cell reduction associated with pioglitazone and fenofibrate. Diabetol. Int., 5, 202-205.

10. Thoolen, B., Maronpot, R.R., Harada, T., Nyska, A., Rousseaux, C., Nolte, T., Malarkey, D.E., Kaufmann, W., Küttler, K., Deschl, U., Nakae, D., Gregson, R., Vinlove, M.P., Brix, A.E., Singh, B., Belpoggi, F. and Ward, J.M. (2010) Proliferative and nonproliferative lesions of the rat and mouse hepatobiliary system. Toxicol. Pathol., 38, 5S-81S.

11. Welte, K. (2014) G-CSF: filgrastim, lenograstim and biological similars. Expert Opin. Biol. Ther., 14, 983-993.

12. Akizuki, S., Mizorogi, F., Inoue, T., Sudo, K. and Ohnishi, A. (2000) Pharmacokinetics and adverse events following 5-day repeated administration of lenograstim, a recombinant human granulocyte colony-stimulating factor, in healthy subjects. Bone Marrow Transplant., 26, 939-946.

13. Stewart, C.A. (1974) Leucocyte alkaline phosphatase in myeloid maturation. Pathology, 6, 287-293.

14. Izumi, M., Ishikawa, J., Takeshita, A. and Maekawa, M. (2005) Increased serum alkaline phosphatase activity originating from neutrophilic leukocytes. Clin. Chem., 51, 1751-1752.

15. Frazier, K.S., Seely, J.C., Hard, G.C., Betton, G., Burnett, R., Nakatsuji, S., Nishikawa, A., Durchfeld-Meyer, B. and Bube, A. (2012) Proliferative and nonproliferative lesions of the rat and mouse urinary system. Toxicol. Pathol., 40, 14S-86S.

16. Kaufmann, W., Bolon, B., Bradley, A., Butt, M., Czasch, S., Garman, R.H., George, C., Gröters, S., Krinke, G., Little, P., McKay, J., Narama, I., Rao, D., Shibutani, M. and Sills, R. (2012) Proliferative and nonproliferative lesions of the rat and mouse central and peripheral systems. Toxicol. Pathol., 40, $87 \mathrm{~S}-157 \mathrm{~S}$.

17. Haschek, W.M., Rousseaux, C.G. and Wallig, M.A. (2010) Fundamentals of toxicologic pathology (2nd edition), Academic Press, San Diego, pp. 1-691.

18. Peter, M. (2012) Background lesions in laboratory animals: a color atlas. Elsevier, New York, pp. 1-256.

19. Hard, G.C. and Khan, K.N. (2004) A contemporary overview of chronic progressive nephropathy in the laboratory rat, and its significance for human risk assessment. Toxicol. Pathol., 32, 171-180. 